Sample: 141

File: Ar29532_41

Vial: $A / 6$
Date: 01-Jul-2009

Time: 02:17:12

Description: 10912004
Page 1.

AMRI code: ALB-H04784390

Vial label: M4756674AMP0007

\title{
(2) ELSD Signal
}

max. intensity: $1 \mathrm{E} 6$

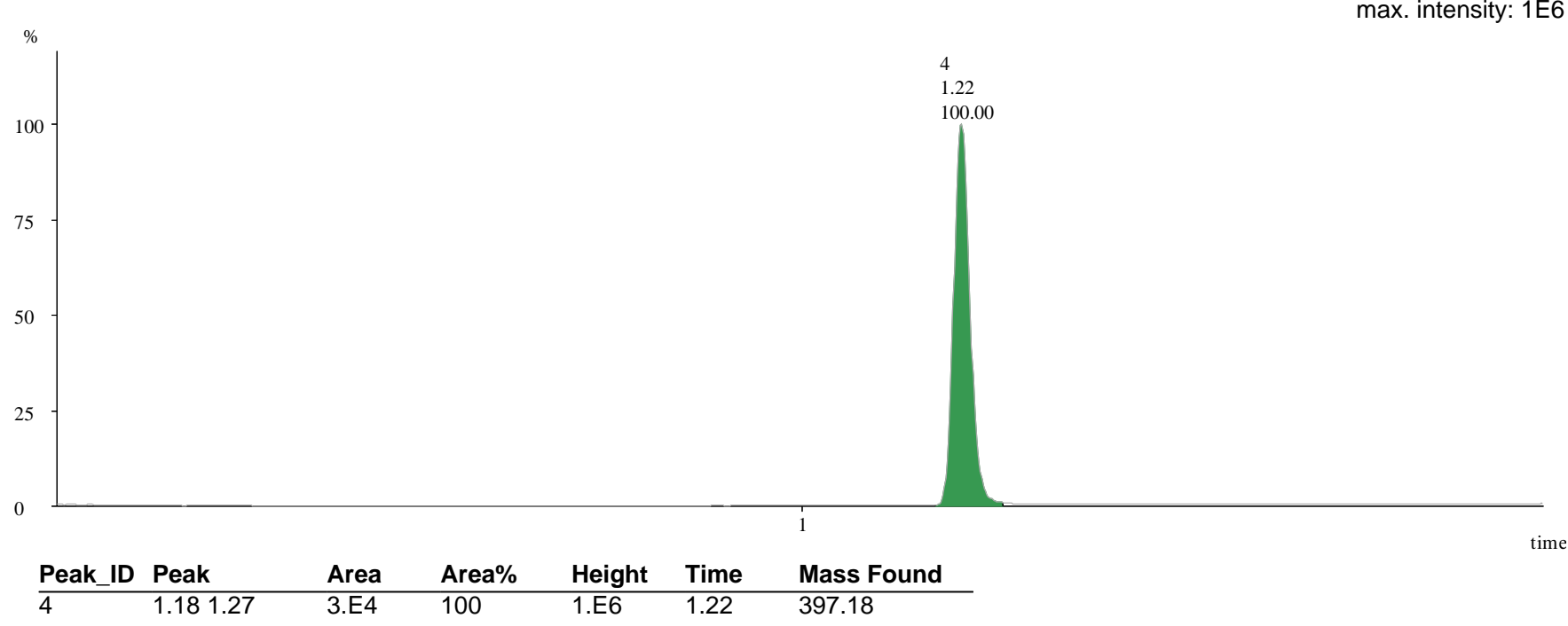

DAD: 220

max. intensity: $2.3 E 6$

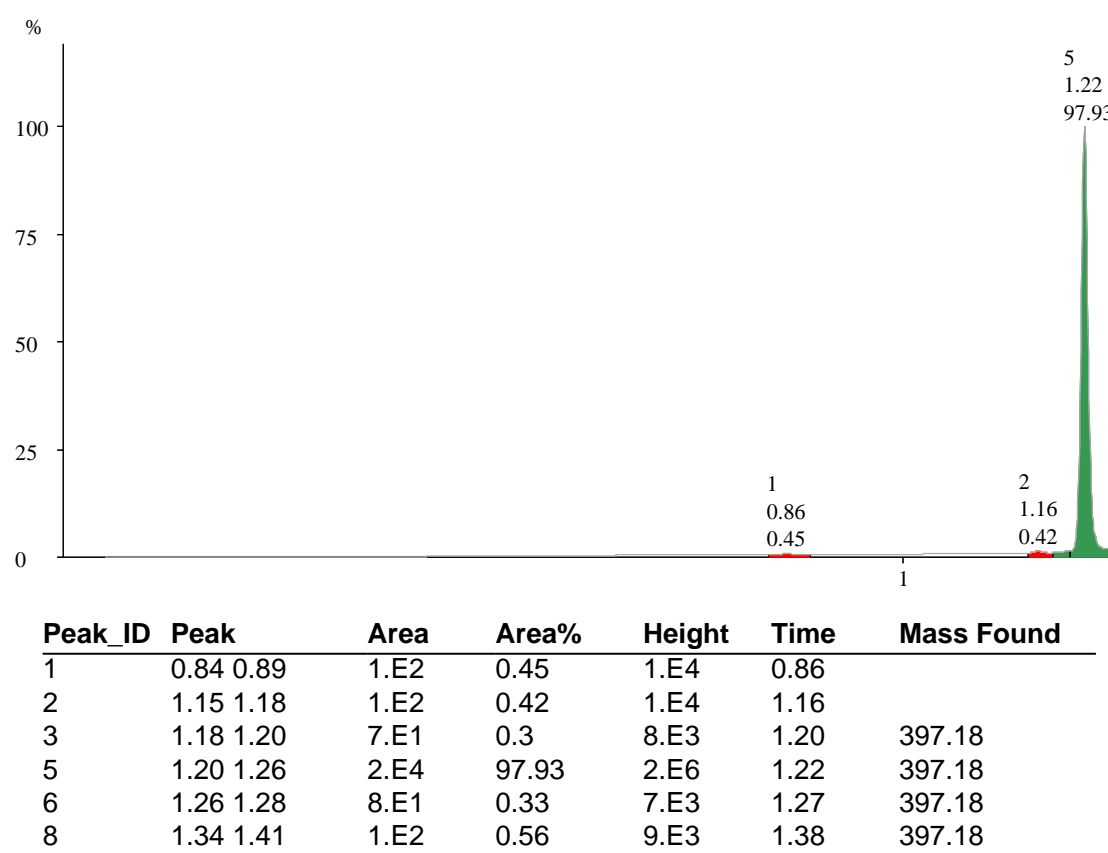


Sample: 141

File: Ar29532_41

Vial: $A / 6$
Date: 01-Jul-2009

Time: 02:17:12

Description: 10912004
Page 2.

AMRI code: ALB-H04784390

Vial label: M4756674AMP0007

MS AP+ :398.18

max. intensity: 3.7E6

$\%$
100
75
50
25
0

0

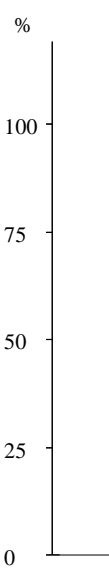

4
1.21
100.00

100.00

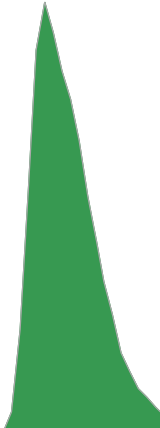

Peak_ID Peak

Area

Area\%

Heigh

Time

Mass Found

time

\section{MS AP+ :TIC}

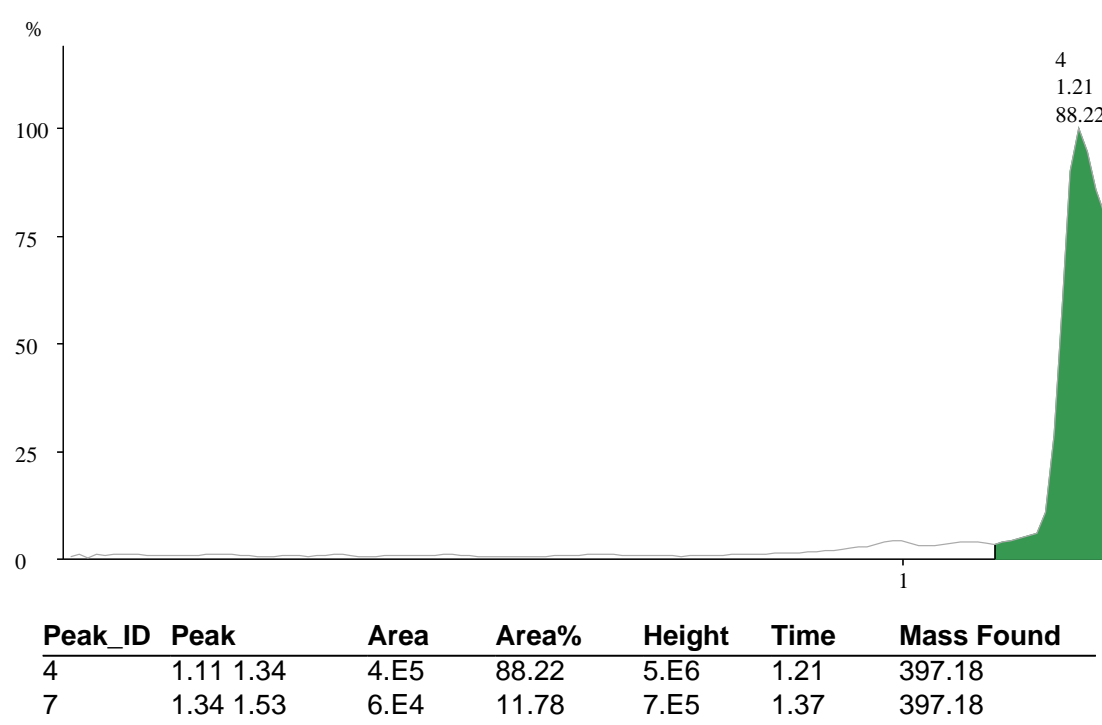

max. intensity: 5E6

\begin{tabular}{lllllll}
\hline 4 & 1.111 .34 & $4 . E 5$ & 88.22 & $5 . E 6$ & 1.21 & 397.18 \\
7 & 1.341 .53 & $6 . E 4$ & 11.78 & $7 . E 5$ & 1.37 & 397.18
\end{tabular}


Sample: 141

File: Ar29532_41

Vial: $A / 6$
Date: 01-Jul-2009

Time: 02:17:12

Description: 10912004
Page 3.

AMRI code: ALB-H04784390

Vial label: M4756674AMP0007

\section{MS: AP+}

Combine (119:122-(112:115+129:132))

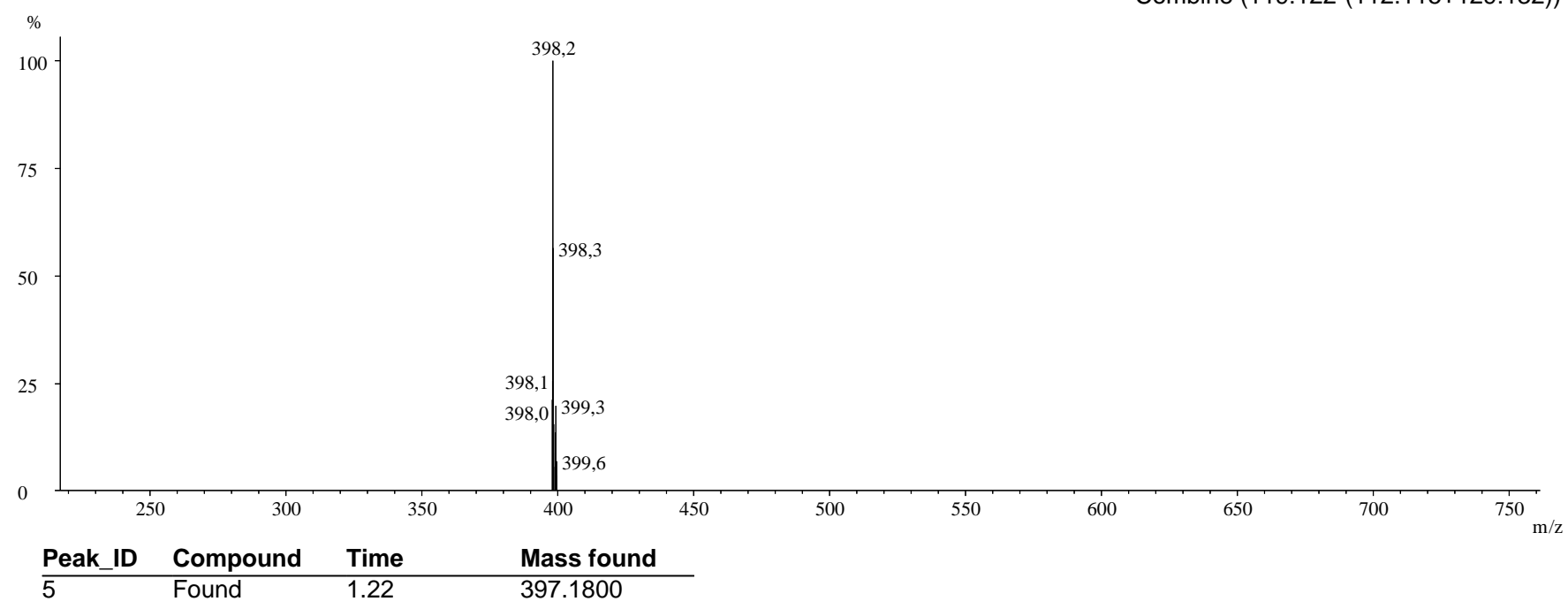




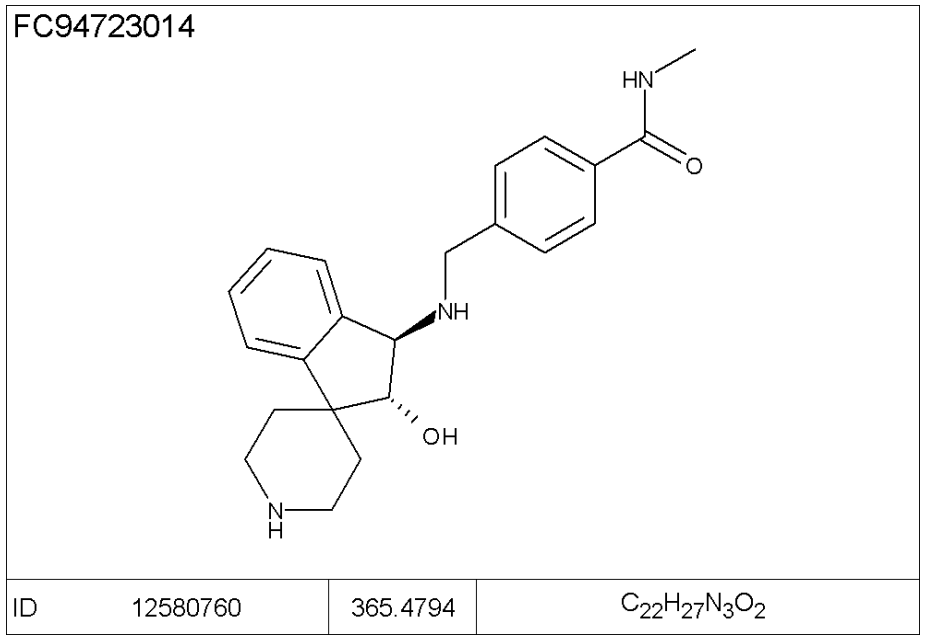

Data File R: \HPLC \AUTO \FC947230\2FB-2001.D

Sample Name: FC947230P2-F-02

Instrument 1 02/03/2012 15:06:54

PMP1, Solvent A : $0.1 \% \mathrm{TFA}$ in $\mathrm{Acn} / \mathrm{H} 2 \mathrm{O}(2.5: 97.5)$

PMP1, Solvent B : $0.1 \% \mathrm{TFA}$ in $\mathrm{AcN}$

PMP1, Solvent $C \quad: 0.1 \% \mathrm{FA}$ in $\mathrm{ACN} / \mathrm{H} 2 \mathrm{O}(2.5: 97.5)$

PMP1, Solvent D : $0.1 \% \mathrm{FA}$ in $\mathrm{ACN}$

Ionization mode : API-ES Positive

Signal 1: ADC1 A, ELSD

\begin{tabular}{|c|c|c|c|c|c|c|}
\hline $\begin{array}{c}\text { Peak } \\
\text { \# }\end{array}$ & $\begin{array}{l}\text { RetTime } \\
\text { [min] }\end{array}$ & Type & $\begin{array}{l}\text { Width } \\
\text { [min] }\end{array}$ & $\begin{array}{r}\text { Area } \\
{\left[\mathrm{mV}^{\star} \mathrm{s}\right]}\end{array}$ & $\begin{array}{l}\text { Height } \\
{[\mathrm{mV}]}\end{array}$ & $\begin{array}{c}\text { Area } \\
\frac{\circ}{0}\end{array}$ \\
\hline 1 & 0.756 & MM & 0.0422 & 318.94 & 125.840 & 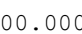 \\
\hline
\end{tabular}

Totals :

$318.94034 \quad 125.84002$

Signal 2: DAD1 A, Sig=300,200 Ref=off

\begin{tabular}{|c|c|c|c|c|c|c|}
\hline $\begin{array}{c}\text { eak } \\
\text { \# }\end{array}$ & $\begin{array}{c}\text { RetTime } \\
\text { [min] }\end{array}$ & Type & $\begin{array}{l}\text { Width } \\
\text { [min] }\end{array}$ & $\begin{array}{c}\text { Area } \\
{\left[\mathrm{mAU}^{*} \mathrm{~S}\right]}\end{array}$ & $\begin{array}{l}\text { Height } \\
\text { [mAU] }\end{array}$ & $\begin{array}{c}\text { Area } \\
\frac{\circ}{0}\end{array}$ \\
\hline 1 & 0.69 & & 0.0 & 617 & & \\
\hline otal & $S$ & & & 617. & 166.4 & \\
\hline
\end{tabular}

Signal 3: MSD1 TIC, MS File

\begin{tabular}{|c|c|c|c|c|c|c|}
\hline $\begin{array}{c}\text { Peak } \\
\text { \# }\end{array}$ & $\begin{array}{c}\text { RetTime } \\
\text { [min] }\end{array}$ & Type & $\begin{array}{c}\text { Width } \\
\text { [min] }\end{array}$ & Area & Height & $\begin{array}{c}\text { Area } \\
\frac{\circ}{0}\end{array}$ \\
\hline 1 & 0.737 & MM & 0.06 & 623 & 12 & 00. \\
\hline
\end{tabular}

Totals : $\quad 4.36238 \mathrm{e} 6 \quad 1.12439 \mathrm{e} 6$
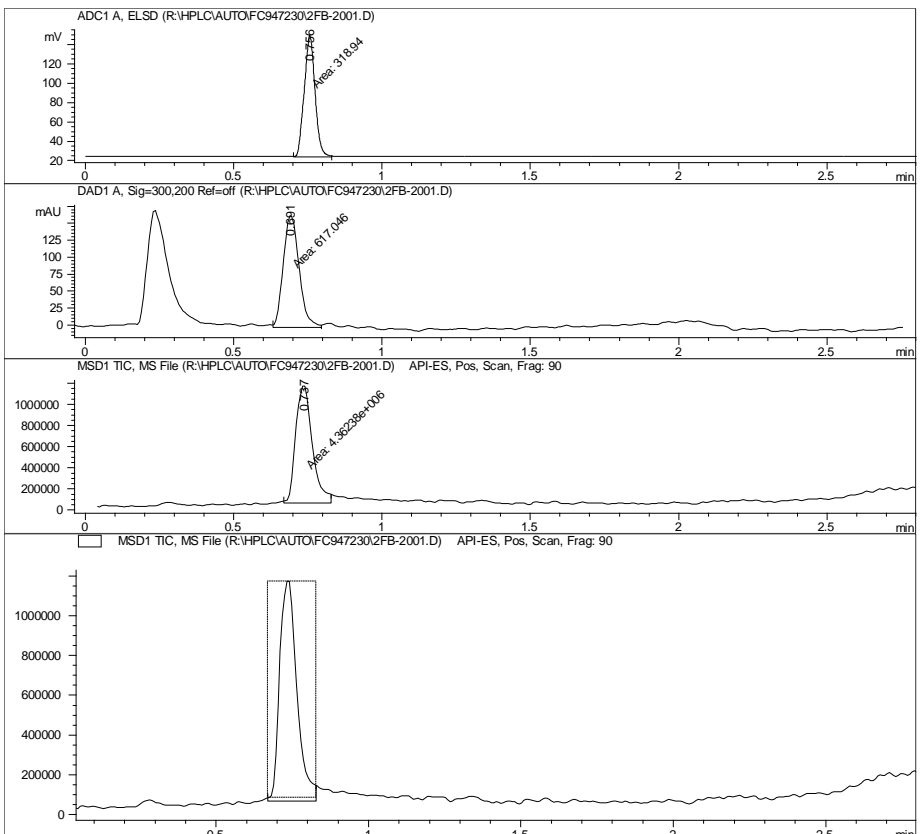

${ }^{*} \mathrm{MSD1}$ SPC, time $=0.673: 0.831$ of R:IIPLCIAUTOIFC94723012FB-2001.

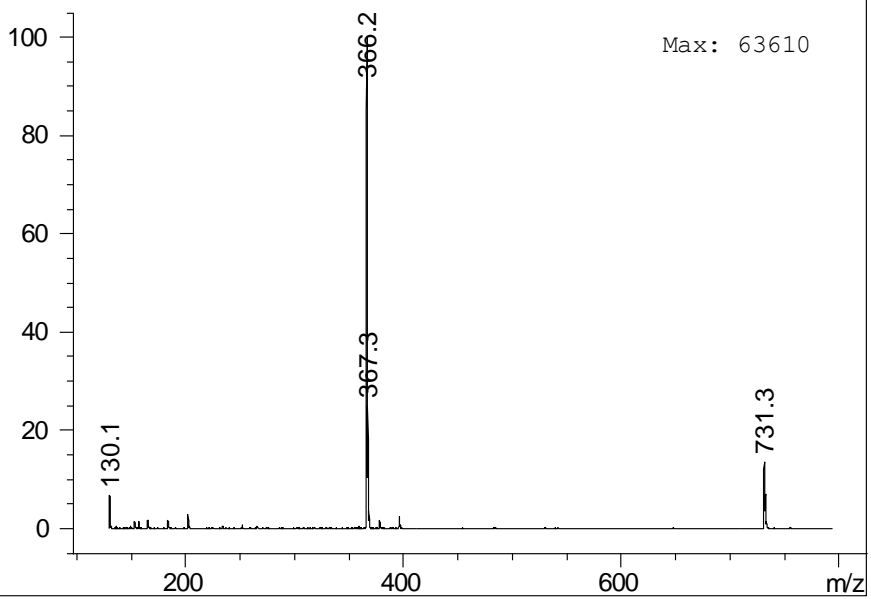




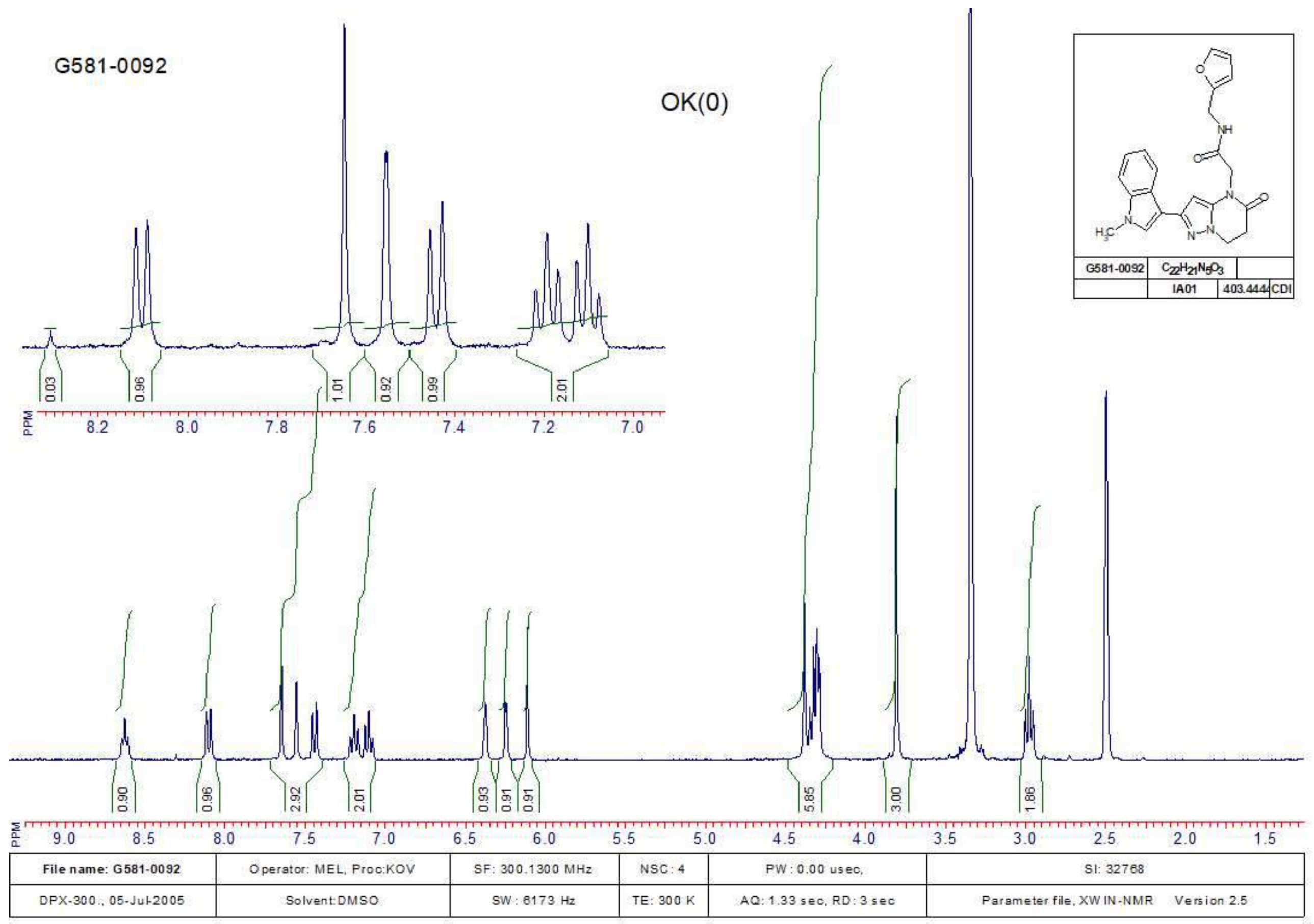




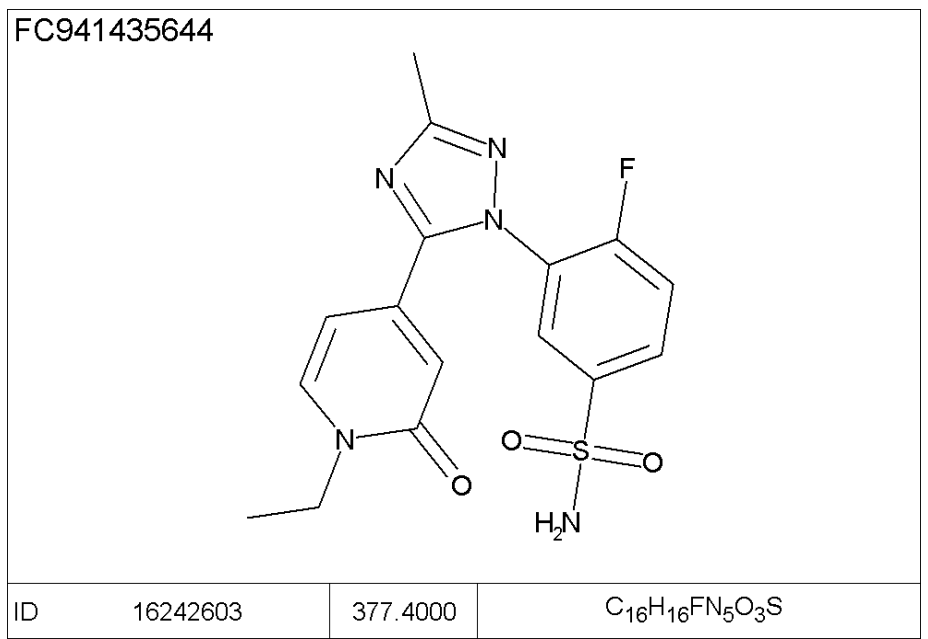

Data File D: \FC9414 1 $\backslash 2 \mathrm{DF}-4401$. D

Sample Name: FC9414356P2-D-06

Instrument 1 21/01/2014 18:27:26

Column: Onyx C18 50x4.6mm | 3.75ml/min | Columns Reg Valve

Gradient: "A"->C2.2min->"B" (Hold $0.4 \mathrm{~min}$ ) ->00.2min->"A"->PostRun

PMP1, Solvent A : $0.1 \% \mathrm{TFA}, 2.5 \circ \mathrm{ACN} / \mathrm{W}$

PMP1, Solvent B : $0.1 \% \mathrm{TFA} / \mathrm{ACN}$

PMP1, Solvent C : --NOT USED--

PMP1, Solvent D : $\mathrm{MeOH}$

Ionization mode : API-ES Positive

Signal 1: ADC1 A, ELSD

Peak RetTime Type Width Area Height Area

$\left[\mathrm{mV}^{*} \mathrm{~s}\right] \quad[\mathrm{mV}] \quad \frac{0}{2}$

1-------|----|-------|----------|----------1-------1

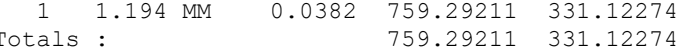

Signal 2: DAD1 A, Sig=300,200 Ref=off

Peak RetTime Type Width Area Height Area

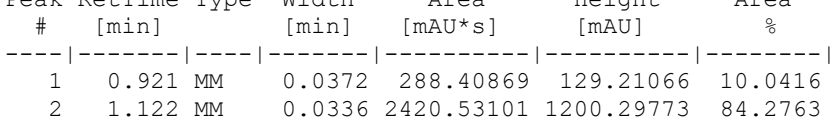

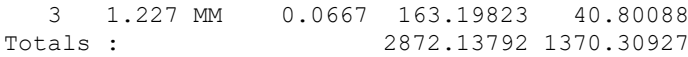

Signal 3: MSD1 TIC, MS File

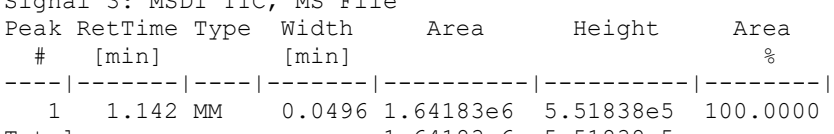

Totals : $\quad 1.64183 \mathrm{e} 6 \quad 5.51838 \mathrm{e} 5$
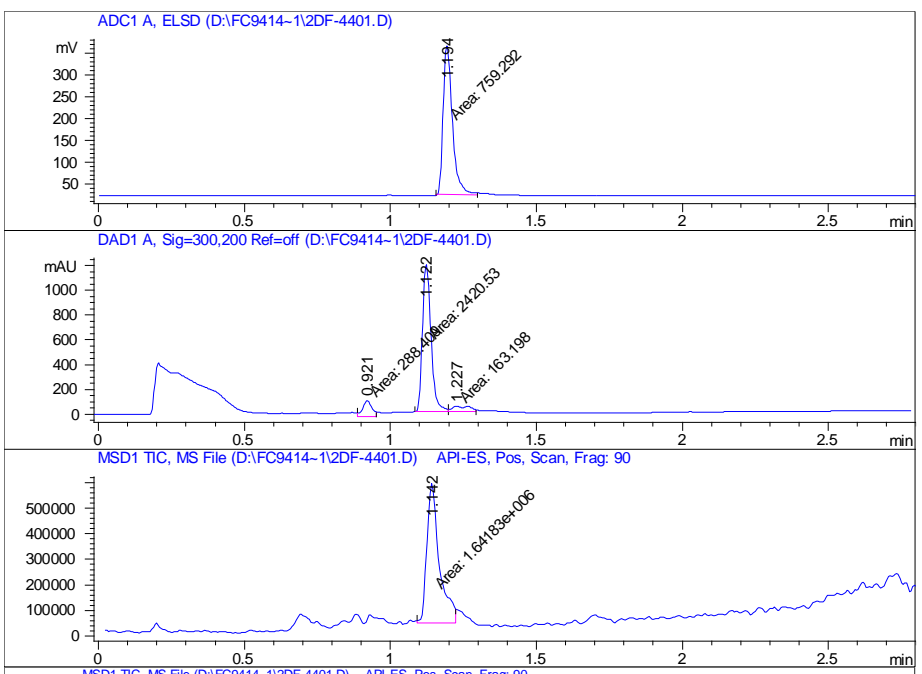

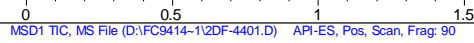

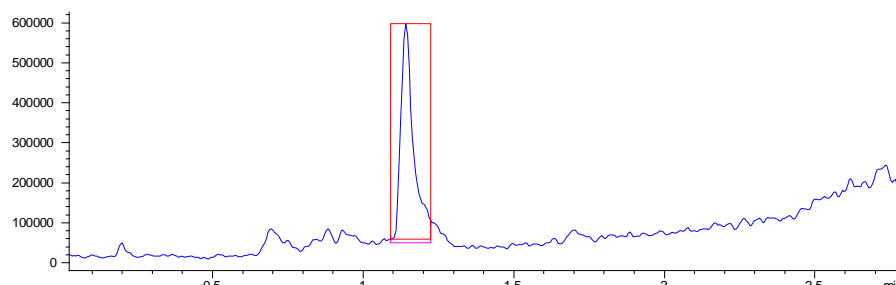

${ }^{*}$ MSD1 SPC time $=1.092: 1.224$ of D:IFC9414 112DF-4401.D API-E

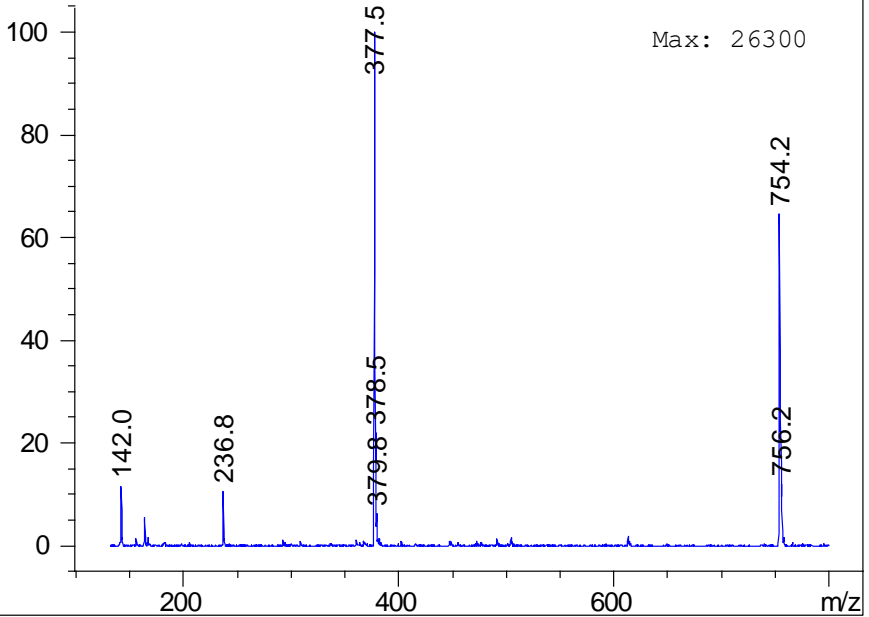


Sample: 18

File: Ar24090_23

Vial: $\mathrm{H} / 2$
Date: 03-Jun-2008

Time: 16:14:53

Description: 20458703
Page 1.

AMRI code: ALB-H11287203

Vial label: 300000245652

\section{(1) ELSD Signal}

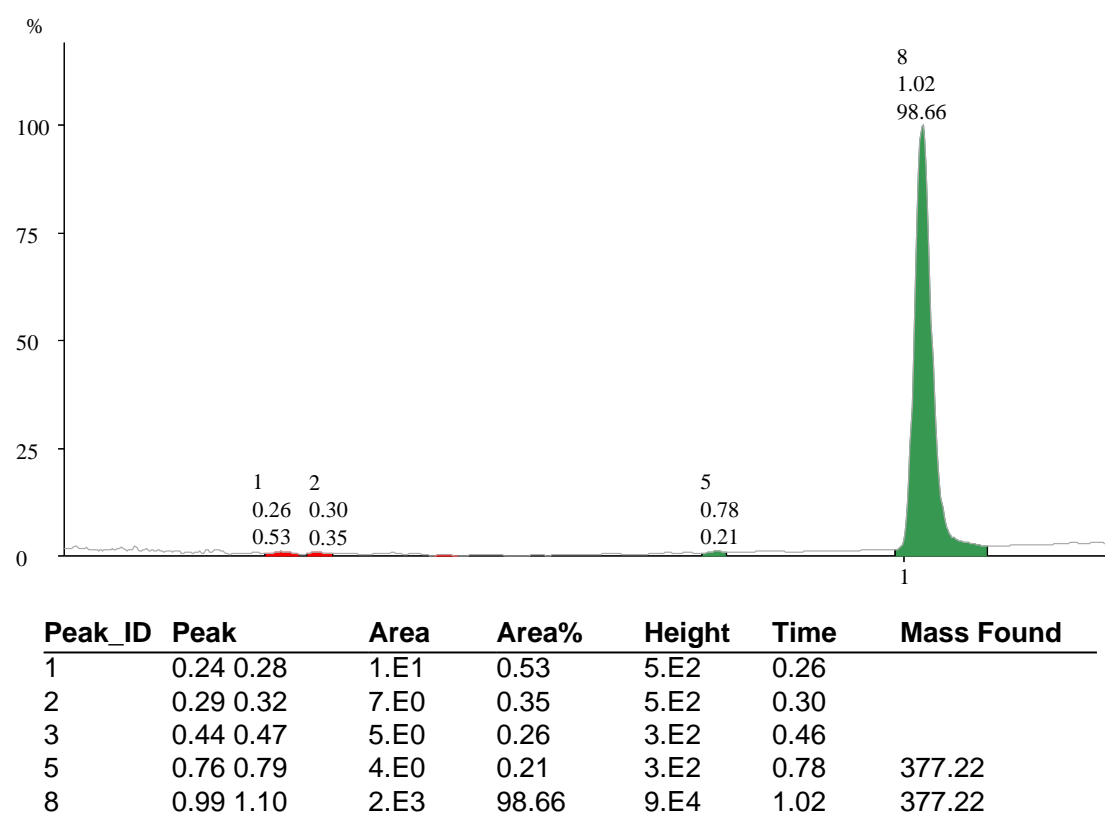

DAD: 220

max. intensity: $2.5 \mathrm{E} 6$

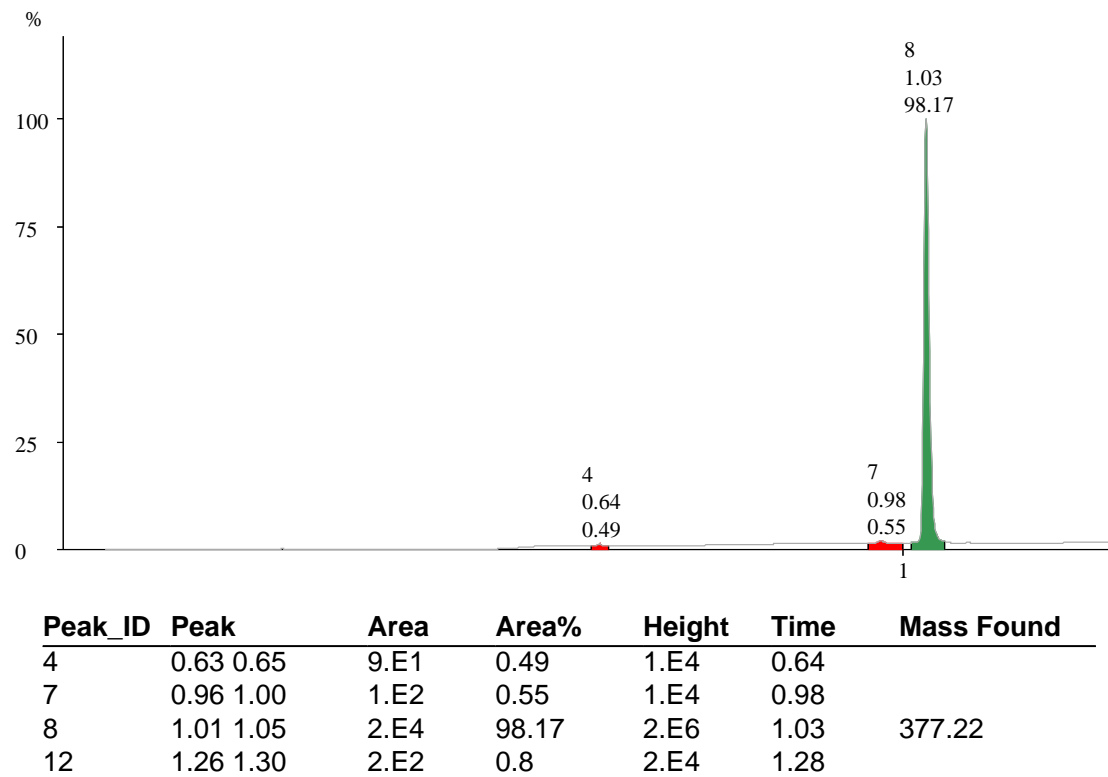


Sample: 18

File: Ar24090_23

Vial: $\mathrm{H} / 2$
Date: 03-Jun-2008

Time: 16:14:53

Description: 20458703
Page 2.

AMRI code: ALB-H11287203

Vial label: 300000245652

MS AP+ :755.44+395.22+378.22

max. intensity: $2.2 \mathrm{E} 7$

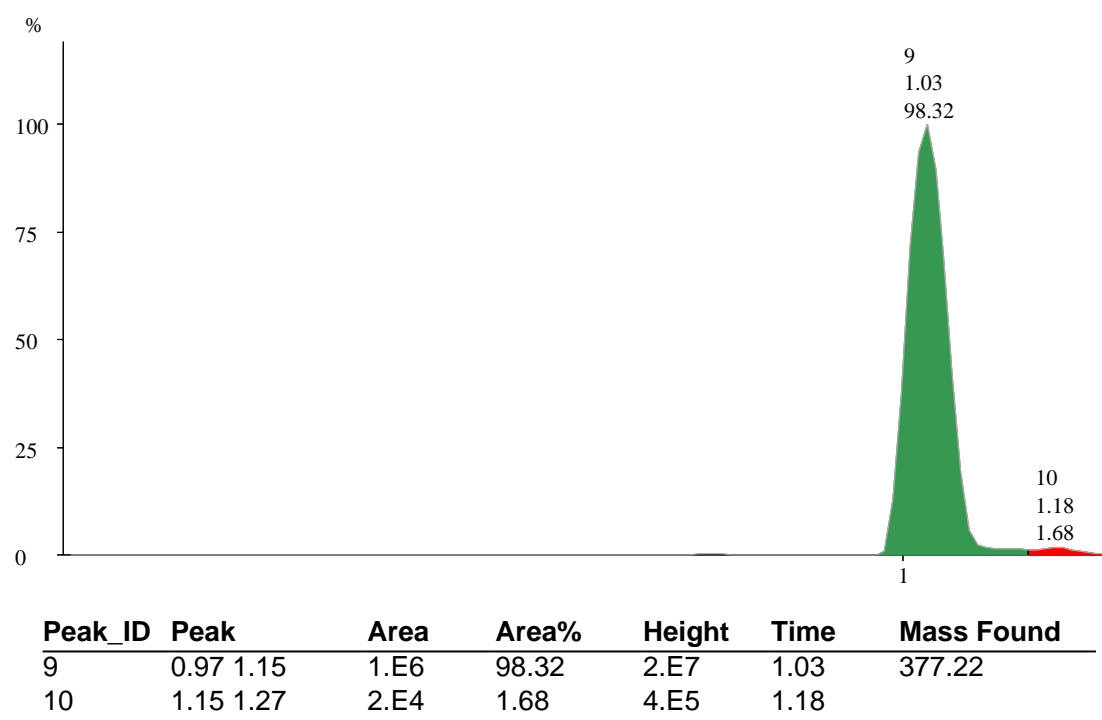

MS AP+ :TIC

max. intensity: $2.8 \mathrm{E} 7$

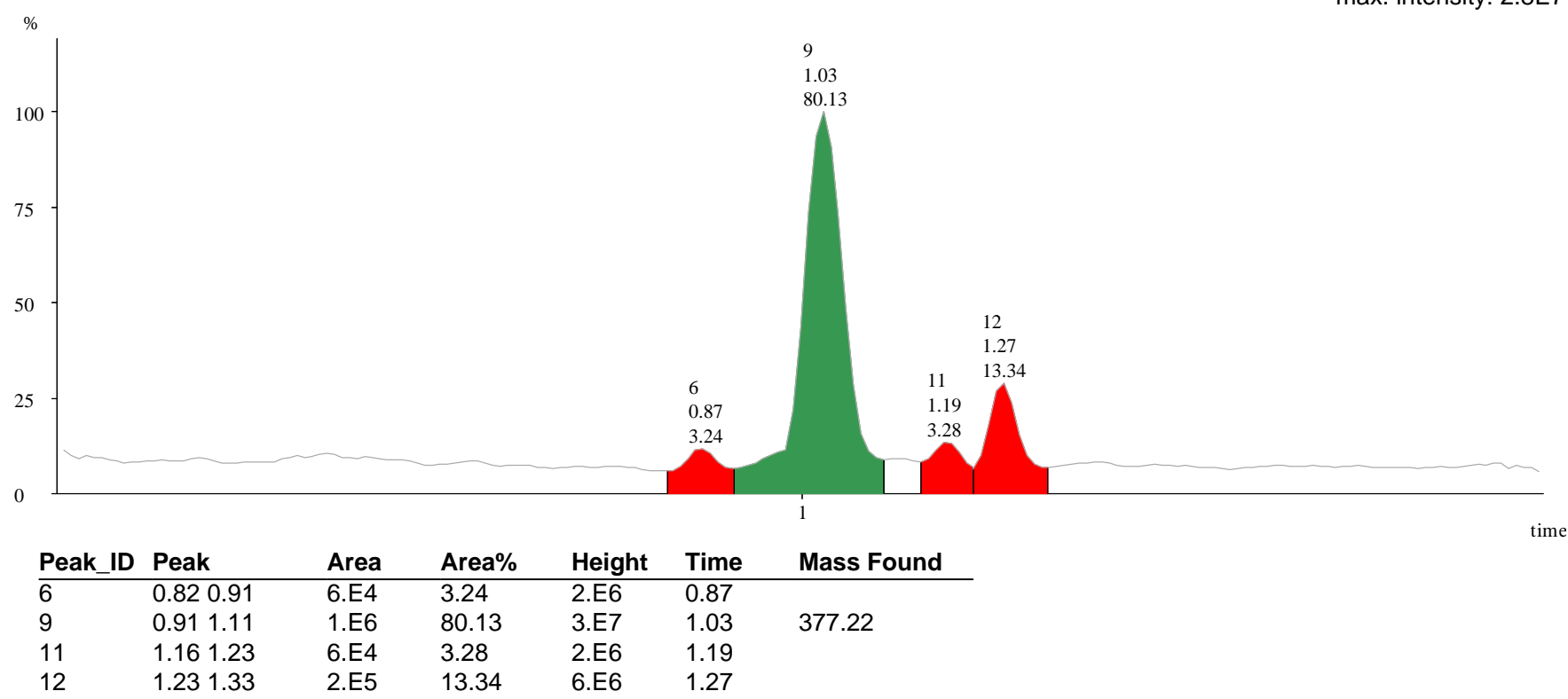


Sample: 18

File: Ar24090_23

Vial: $\mathrm{H} / 2$
Date: 03-Jun-2008

Time: 16:14:53

Description: 20458703
Page 3.

AMRI code: ALB-H11287203

Vial label: 300000245652

\section{MS: AP+}

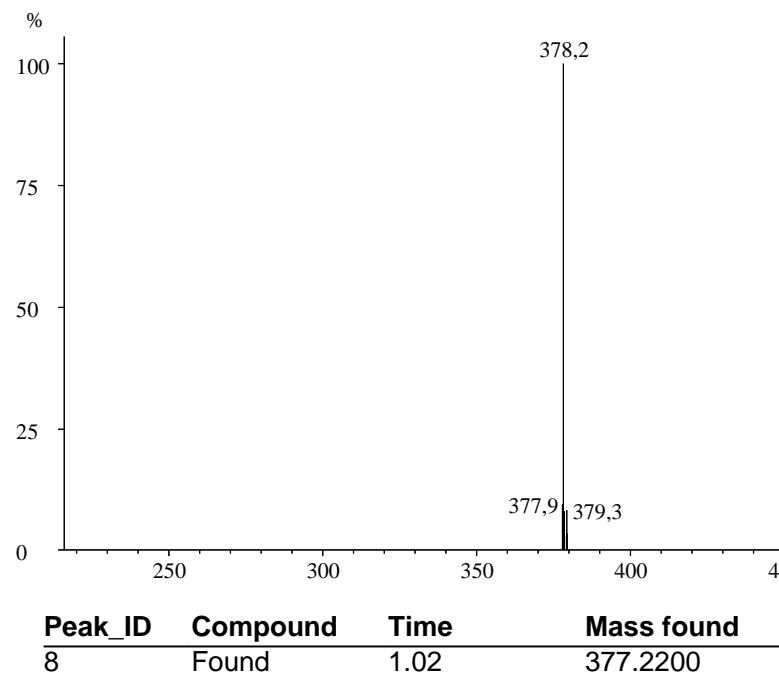




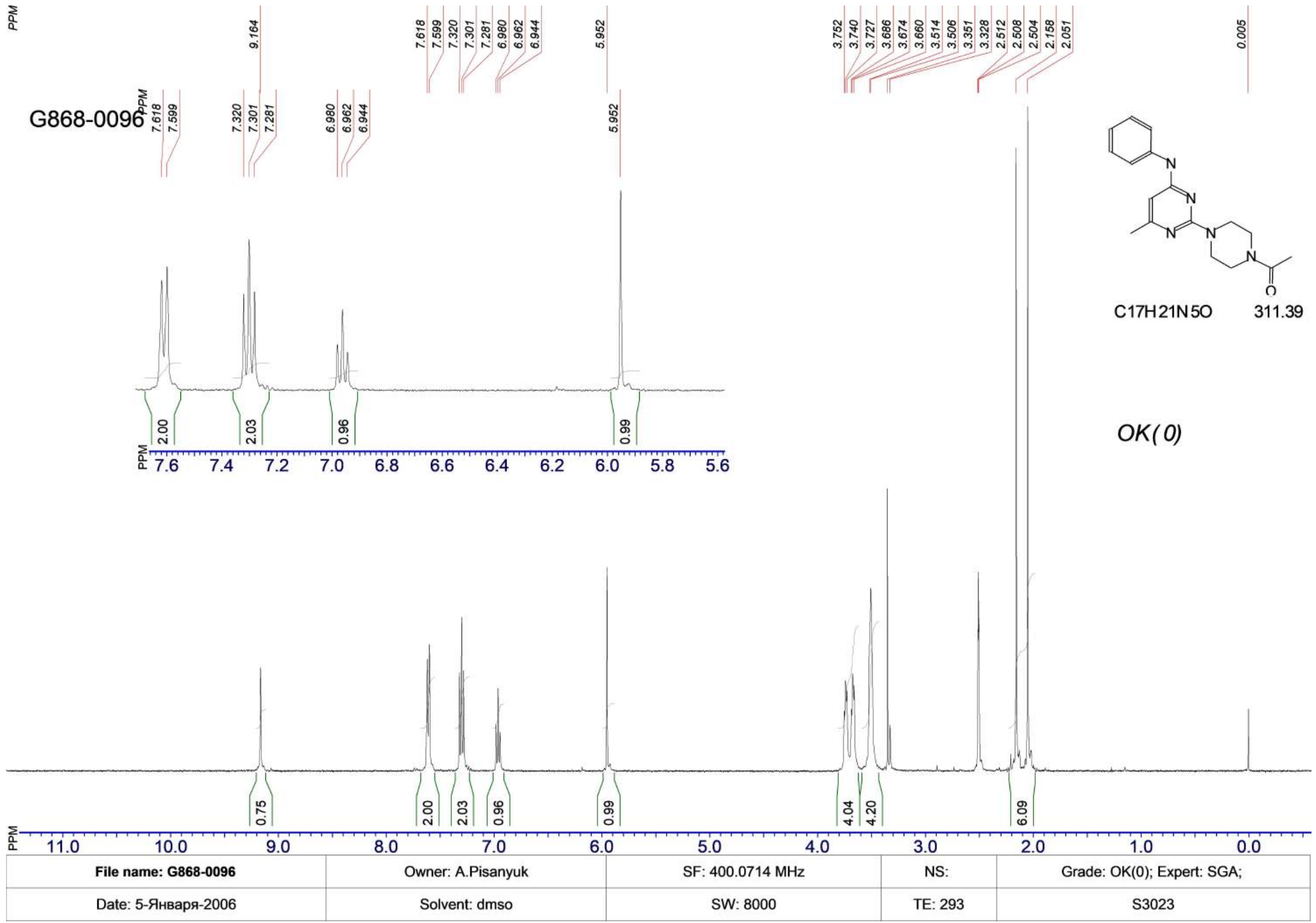


Printed: Mon Dec 08 16:13:20 2003

Sample Report (continued):
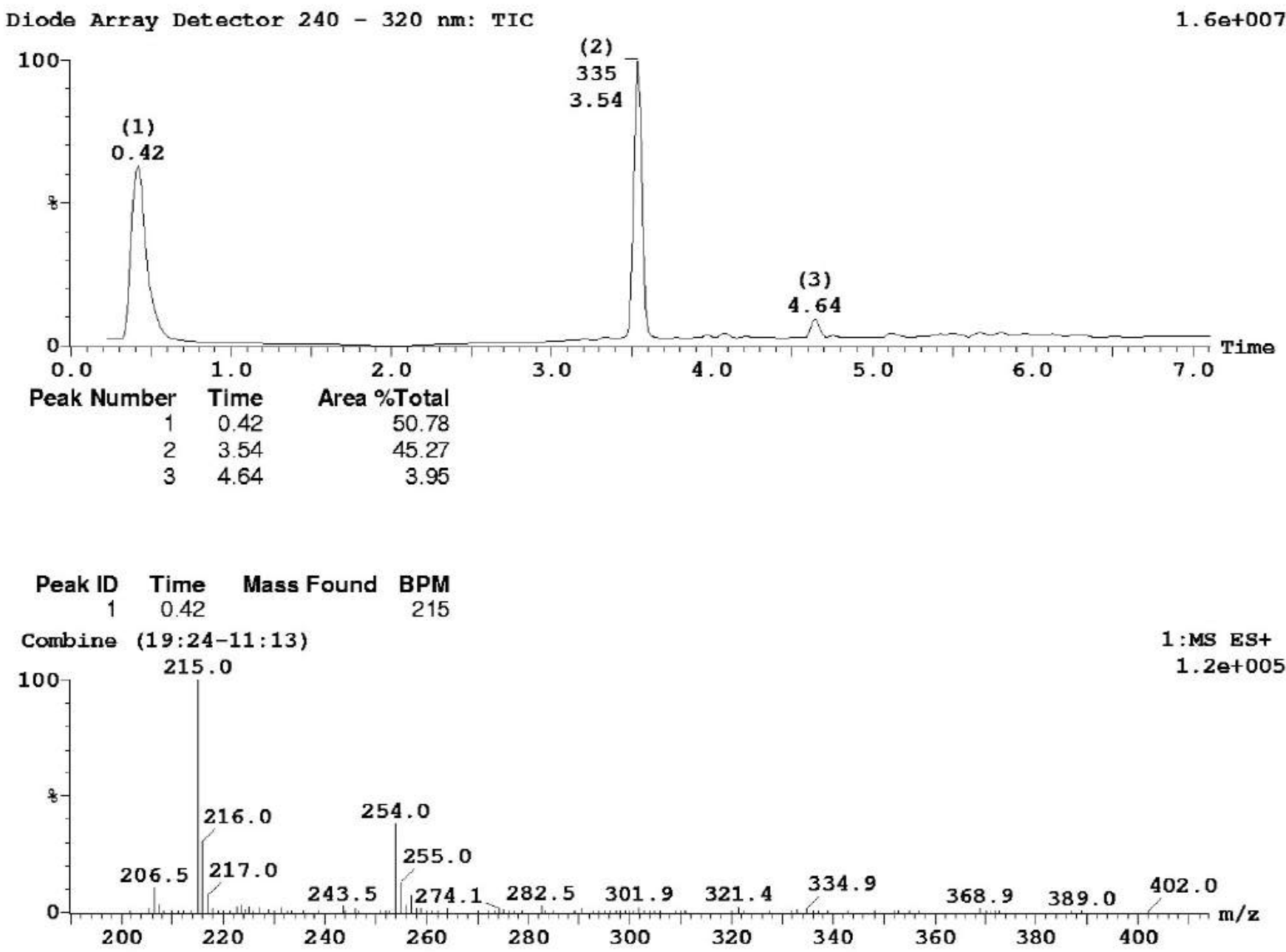

\section{Peak ID Time Mass Found BPM

$2 \quad 3.54 \quad 335.06 \quad 336$

Combine (189:194-179:182)

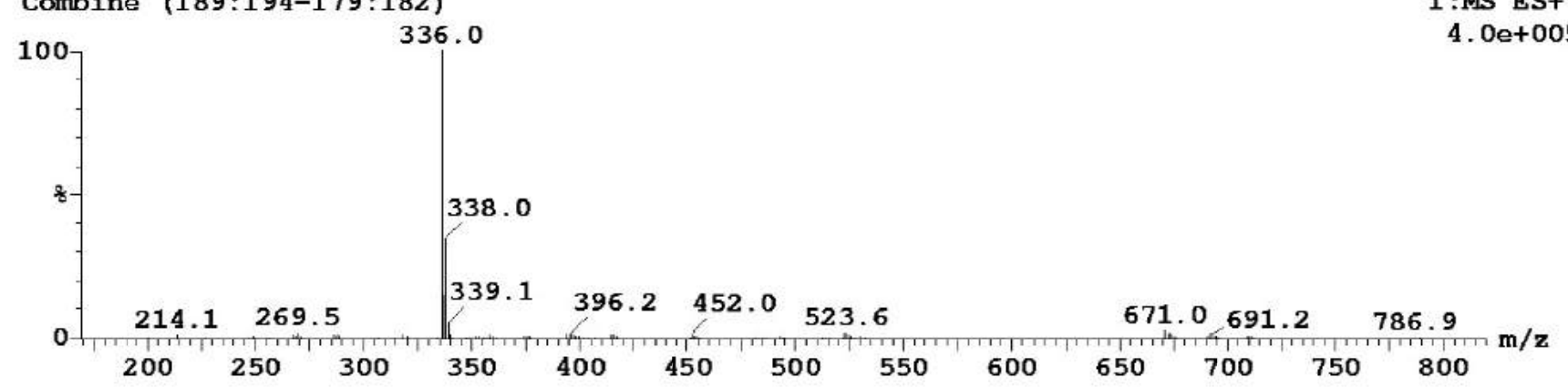


2: UV Detector: TIC

(2)

249.06

2.65

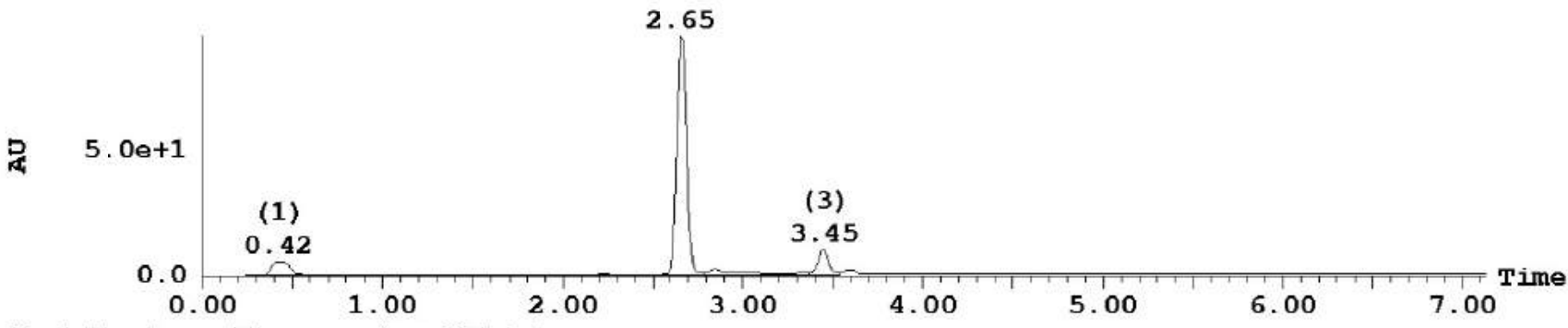

Peak Number Time

$\begin{array}{rrr}1 & 0.42 & \text { Area } \\ 2 & 2.66 & 7.87 \\ 3 & 3.46 & 83.90 \\ & & 8.23\end{array}$

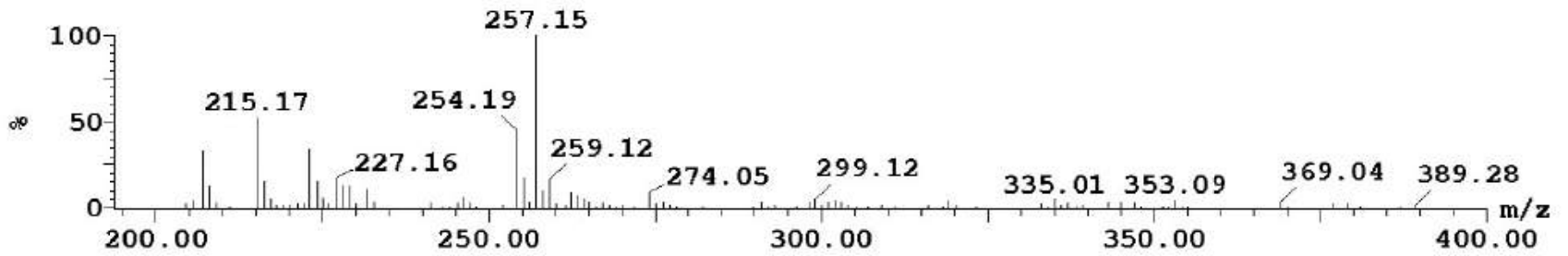

\section{Peak ID Time Mass Found BPM $\begin{array}{llll}2 & 2.66 & 249.06 & 250.19\end{array}$}

Combine (140:147-132:136)
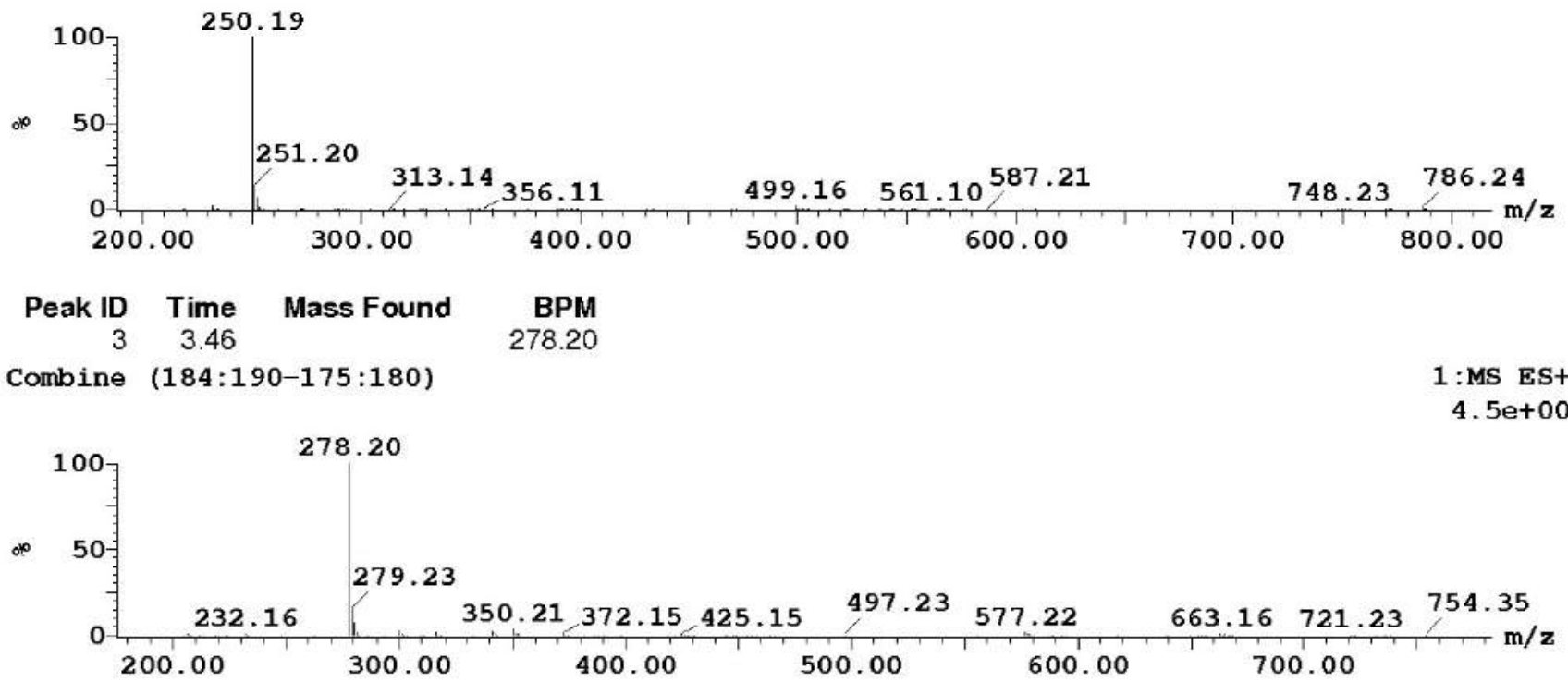
3: UV Detector: TIC
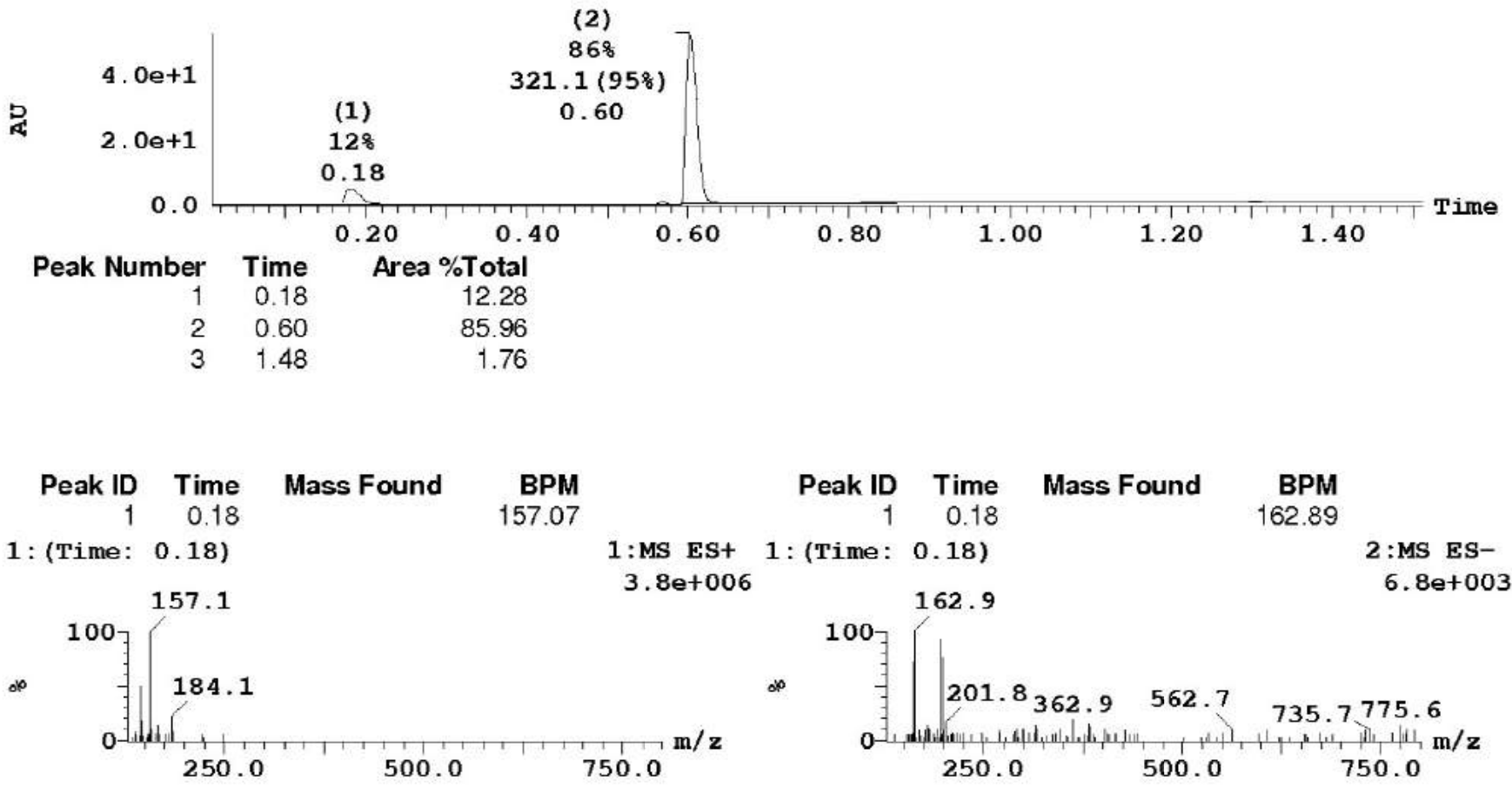

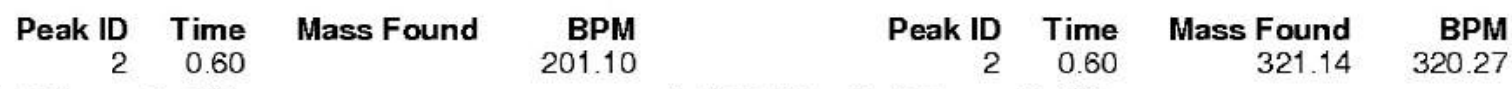

2: (Time: 0.60 )

1:MS ES+ 2: (Time: 0.60 )

2 :MS ES2. $3 e+007$

6. $6 \mathrm{e}+005$
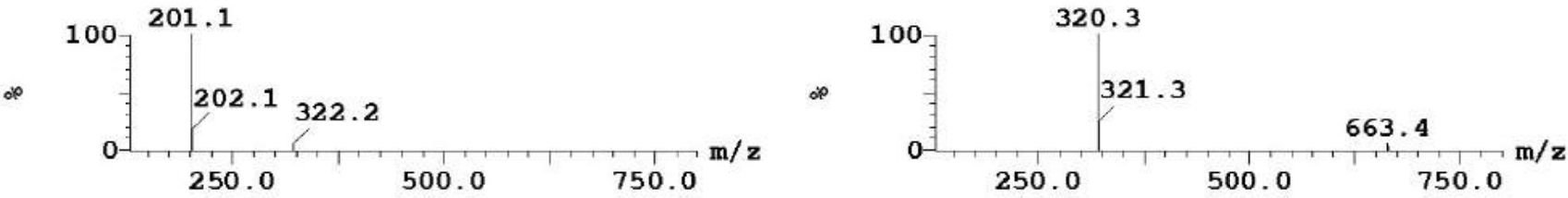

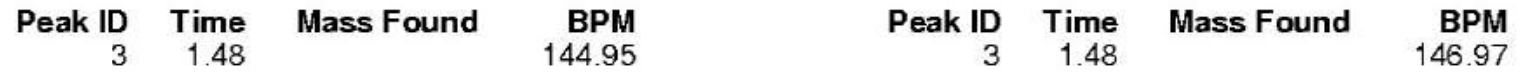

3: (Time: 1.48) 1:MS ES+ 3:(Time: 1.48)

2. $6 e+004$
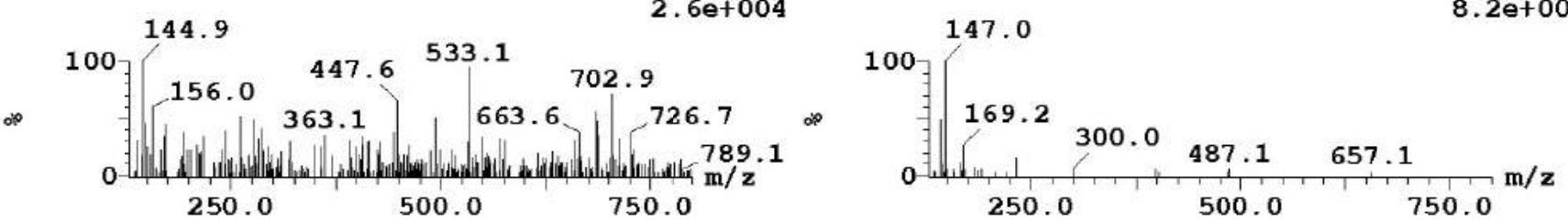
3: UV Detector: TIC

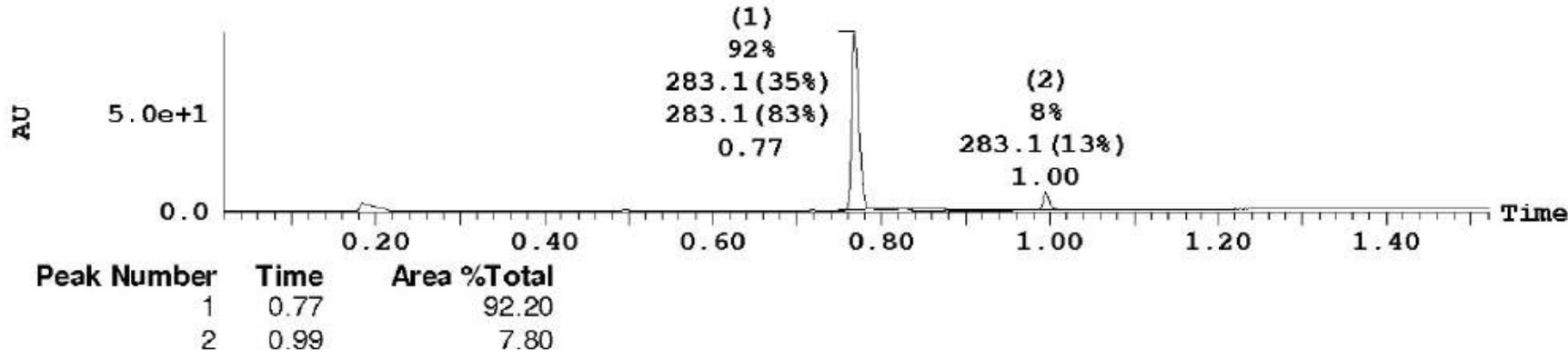

$\begin{array}{rrrrrrrr}\text { Peak ID } & \text { Time } & \text { Mass Found } & \text { BPM } & \text { Peak ID } & \text { Time } & \text { Mass Found } & \text { BPM } \\ 1 & 0.77 & 283.12 & 135.94 & 1 & 0.77 & 283.12 & 282.13\end{array}$

1: (Time: 0.77 )

$1:$ MS ES+ 1:(Time: 0.77 )

2 :MS ES4. $8 e+007$

4. $1 \mathrm{e}+006$
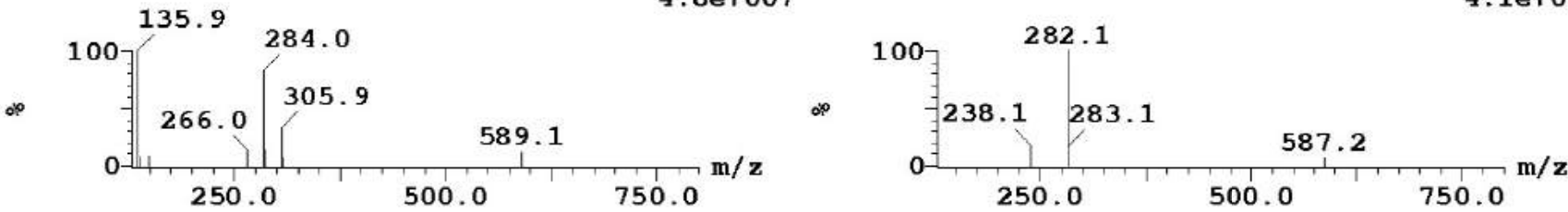

$\begin{array}{rrrrrrrr}\text { Peak ID } & \text { Time } & \text { Mass Found } & \text { BPM } & \text { Peak ID } & \text { Time } & \text { Mass Found } & \text { BPM } \\ 2 & 0.99 & & 265.96 & 2 & 0.99 & 283.12 & 282.10\end{array}$

2: (Time: 0.99 )

1:MS ES+ 2:(Time: 0.99 )

$1.0 e+007$

:MS ES-
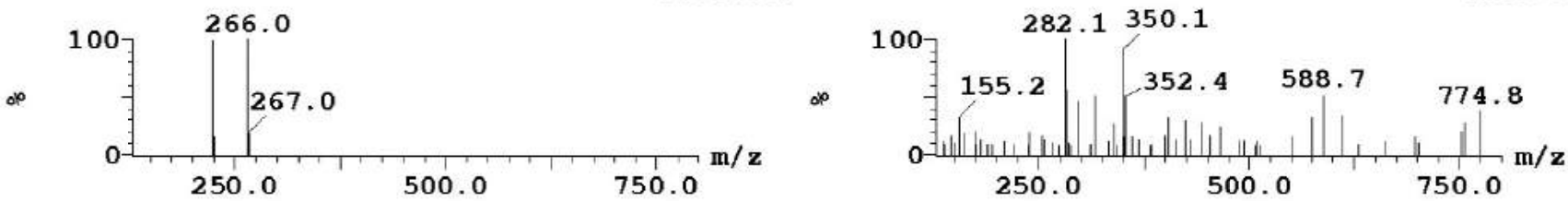
3: UV Detector: TIC

3. $314 \mathrm{e}+1$

Range: $3 \cdot 313 e+1$

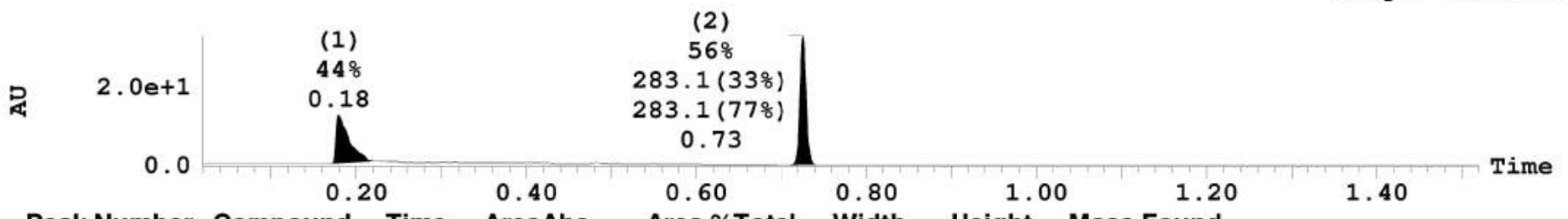

$\begin{array}{rrrrrrrr}\text { Peak Number } & \text { Compound } & \text { Time } & \text { AreaAbs } & \text { Area \%Total } & \text { Width } & \text { Height } & \text { Mass Found } \\ 1 & & 0.18 & 2 \mathrm{e}+005 & 43.76 & 0 & 1 \mathrm{e}+007 & \\ 2 & \text { Found } & 0.73 & 3 \mathrm{e}+005 & 56.24 & 0 & 3 \mathrm{e}+007 & 283.12,283.12\end{array}$

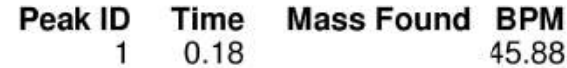

$\begin{array}{rrr}\text { Peak ID } & \text { Time } & \text { Mass Found } \\ 1 & 0.18\end{array} \quad \begin{array}{r}\text { BPM } \\ 45.88\end{array}$

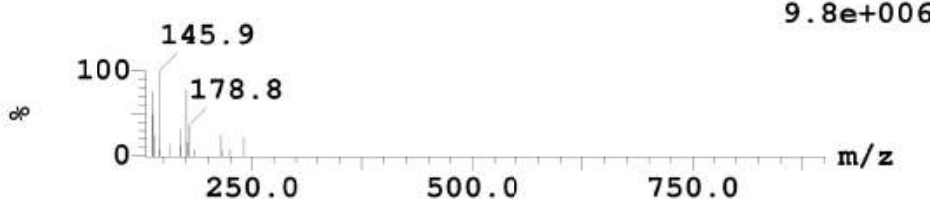

Peak ID Time Mass Found BPM

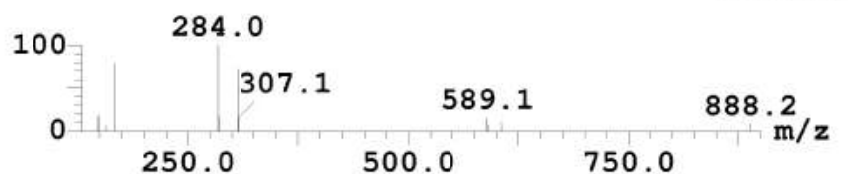

$1:$ MS ES+

2.1e+007

\section{$\begin{array}{rr}\text { Peak ID } & \text { Time } \\ 1 & 0.18\end{array}$ Mass Found $\underset{76.67}{\text { BPM }}$}

\section{$1:$ MS ES+}

:MS ES-

5. $2 \mathrm{e}+002$

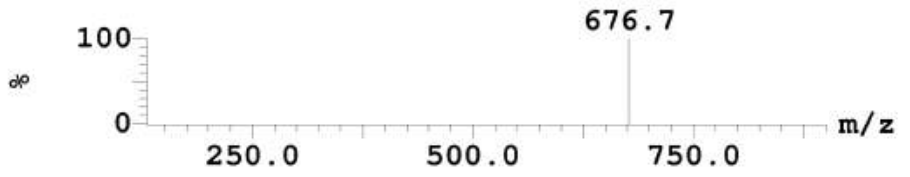

Peak ID Time Mass Found BPM

282.1282 .24

2 :MS ES-

8. $3 e+004$

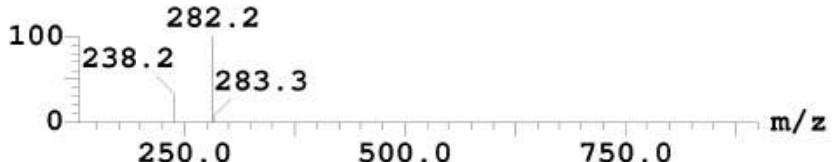


3: UV Detector: TIC

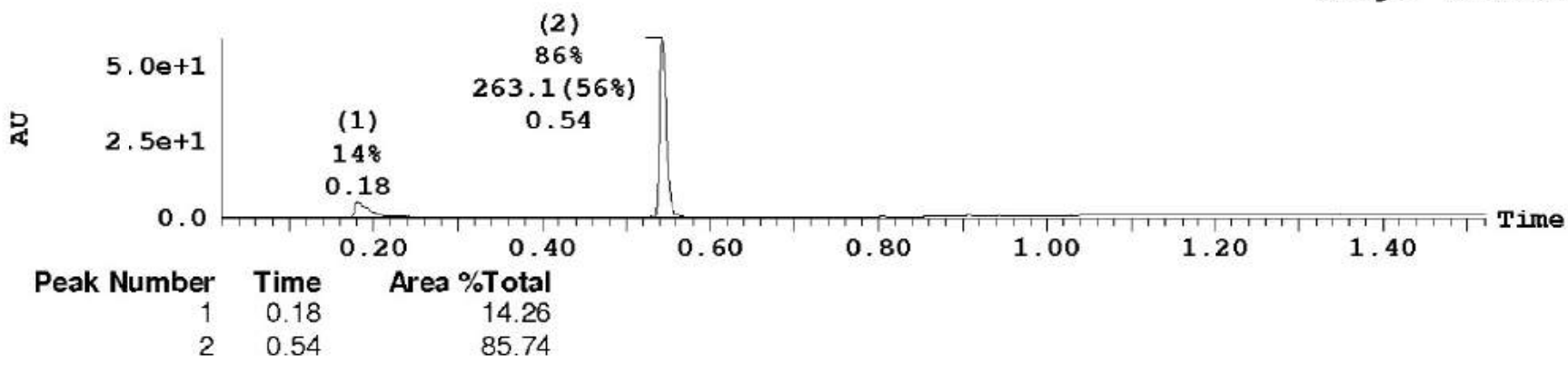

$\begin{array}{rr}\text { Peak ID } & \text { Time } \\ 1 & 0.18\end{array}$ Mass Found $\begin{array}{r}\text { BPM } \\ 175.98\end{array}$

$1:$ (Time: 0.18 )
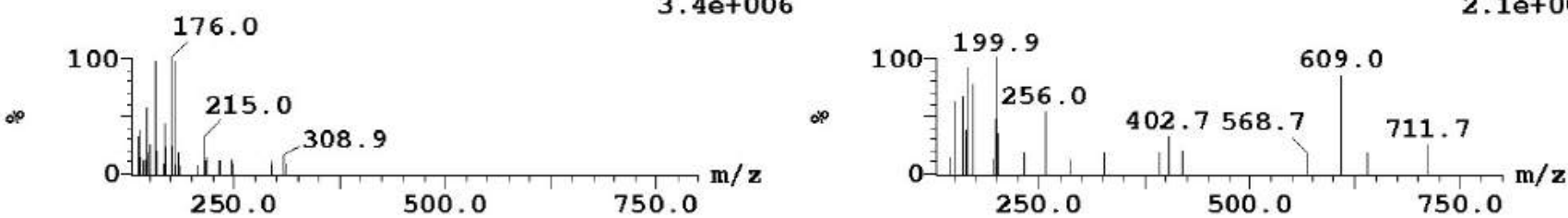

$\begin{array}{rrrr}\text { Peak ID } & \text { Time } & \text { Mass Found } & \begin{array}{r}\text { BPM } \\ 2\end{array} \\ 0.54 & & 175.00 \\ : \text { (Time: } & 0.54 \text { ) }\end{array}$

2: (Time: 0.54 )

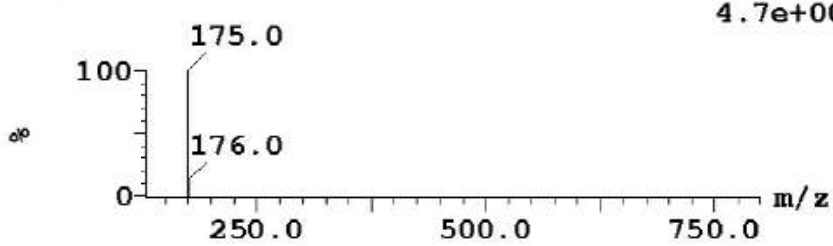

1:MS ES+ 2:(Time: 0.54 )

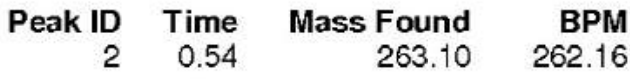

$$
7
$$

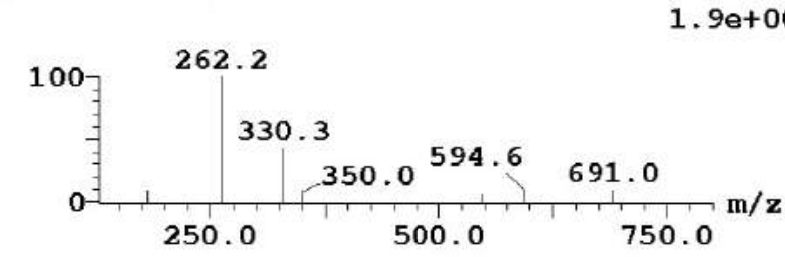


3: UV Detector: TIC Smooth (SG, 2x2)

$2.015 e+2$

Range: $2.014 \mathrm{e}+2$

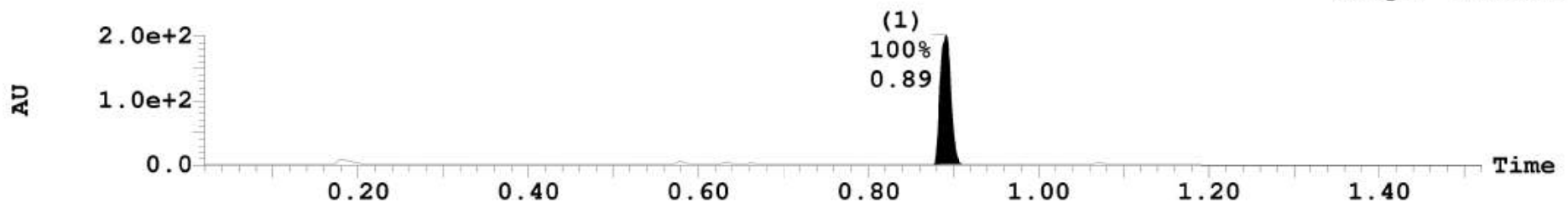

Peak Number Compound Time AreaAbs $1 \quad$ Found $0.89 \quad 3 \mathrm{e}+006$

Area \%Total Width Height Mass Found $\begin{array}{llll}100.00 & 0 & 2 e+008 & 265.11,265.11\end{array}$

\section{Peak ID Time Mass Found BPM 266.1165 .94}

1: (Time: 0.89 )

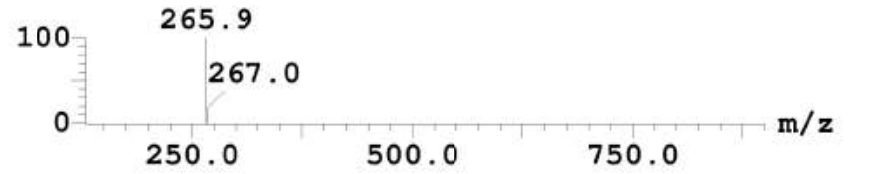

\section{Peak ID Time Mass Found BPM \\ 264.1164 .12}

1:MS ES+ 1: (Time: 0.89 )

2 :MS ES-

7. $7 e+002$

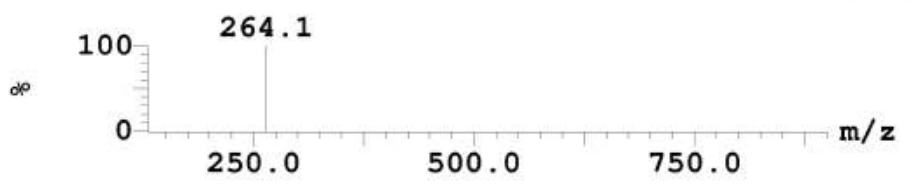


3: UV Detector: TIC

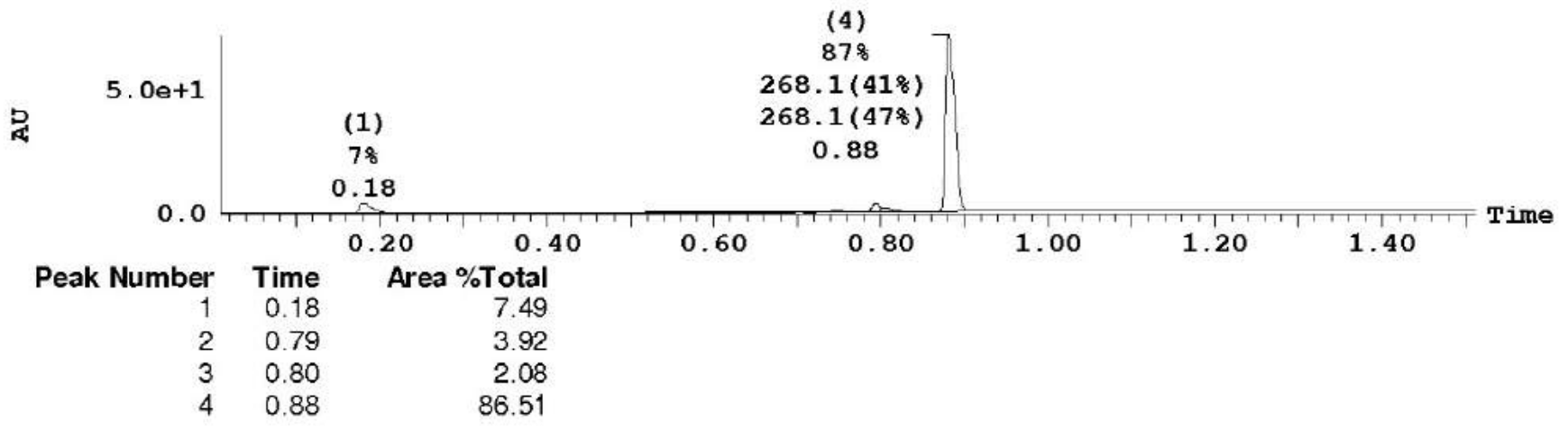

\section{Peak ID Time Mass Found BPM}

Peak ID Time

ime

Mass Found BPM

1: (Time: 0.18 )

$1007 \underbrace{157.0}_{250.0}$

1:MS ES+ 2 : (Time: 0.79 )

1.1e+007 $\frac{1}{10} \mathrm{~m} / \mathrm{z}$

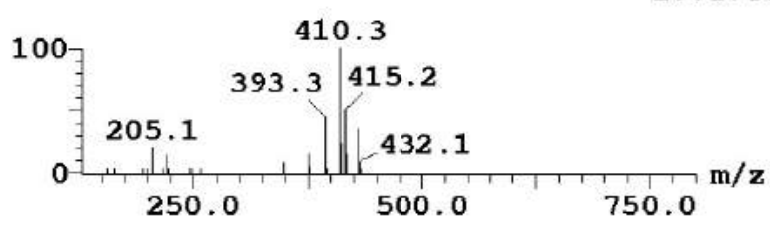

Peak ID Time Mass Found BPM 391.23

$\begin{array}{rr}\text { Peak ID } & \text { Time } \\ 3 & 0.80\end{array}$

2 :MS ES- 3 : (Time: 0.80 )

1. $2 \mathrm{e}+005$

2: (Time: 0.79 )

391.2
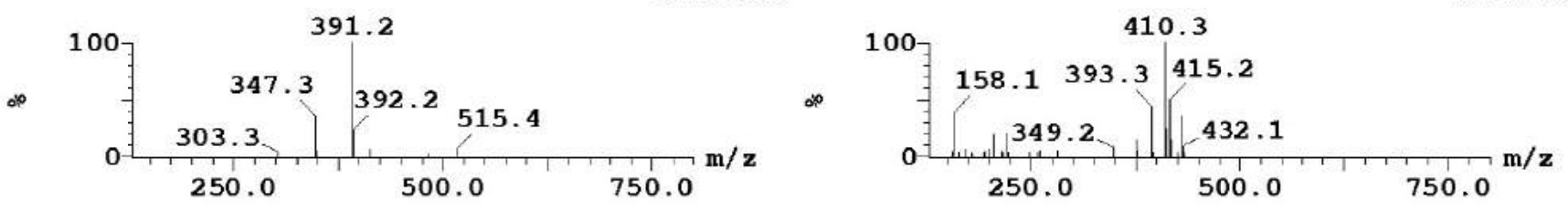

$1:$ MS ES+

. $7 e+005$

4: (Time: 0.88 )

\section{BPM}

Peak ID Time

Mass Found 268.07

BPM

267.21

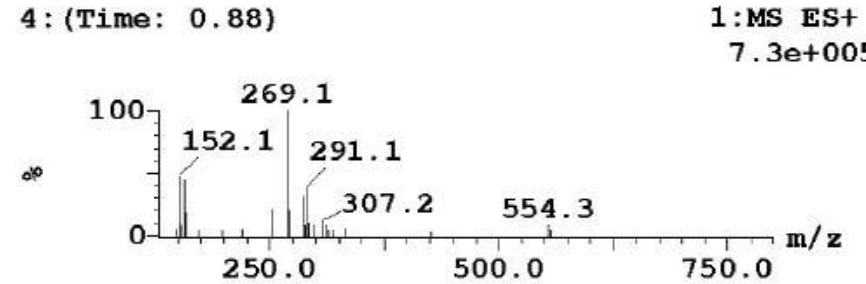

4: (Time: 0.88 )

2 :MS ES-
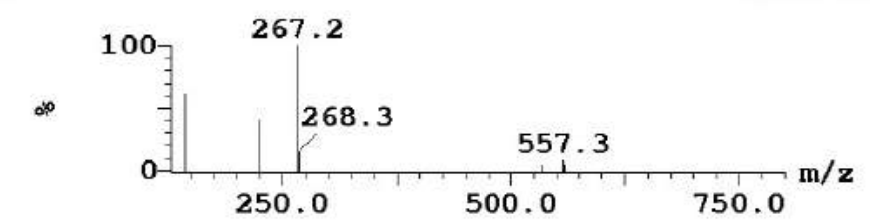
Printed: Mon Apr 07 12:27:13 2003

Sample Report (continued):

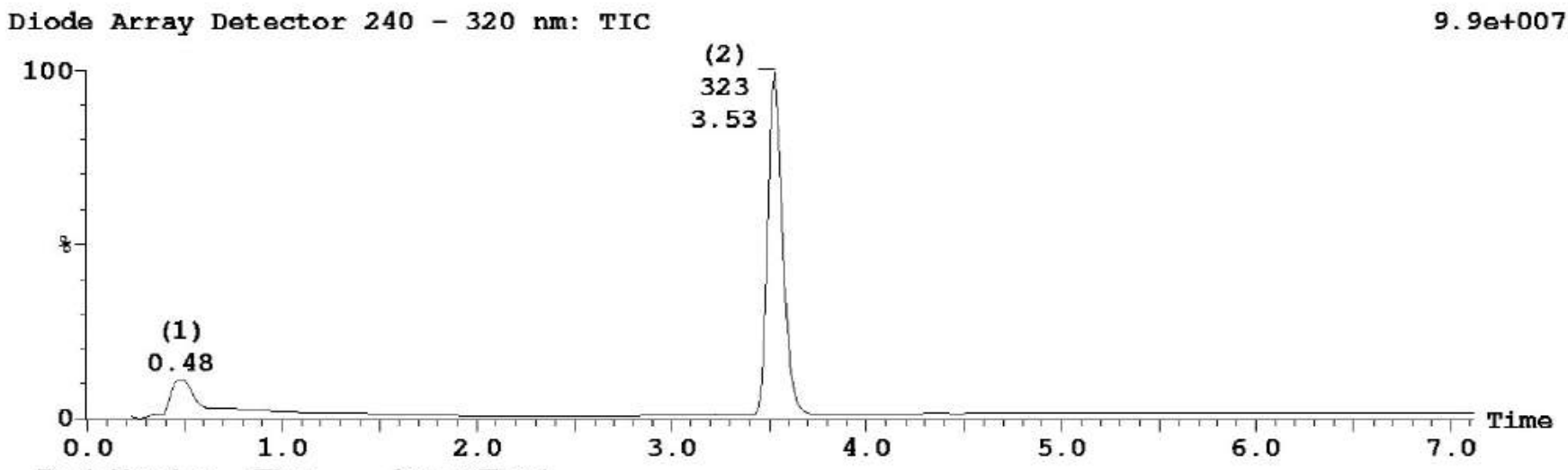

\section{$\begin{array}{rrrr}\text { Peak ID } & \text { Time } & \text { Mass Found } & \text { BPM } \\ 1 & 0.48\end{array}$}

Combine $(22: 28-8: 11)$

$100 \quad 215.3$

1. $5 e+003$

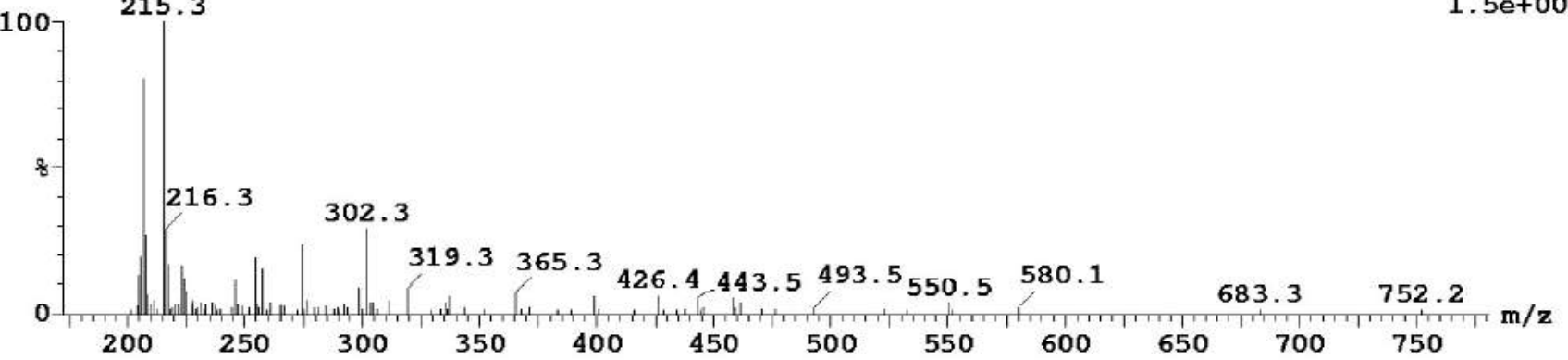

\begin{tabular}{|c|c|c|c|}
\hline $\begin{array}{r}\text { Peak ID } \\
2\end{array}$ & $\begin{array}{r}\text { Time } \\
353\end{array}$ & $\begin{array}{r}\text { Mass Found } \\
32308\end{array}$ & $\begin{array}{r}\text { BPN } \\
322\end{array}$ \\
\hline
\end{tabular}

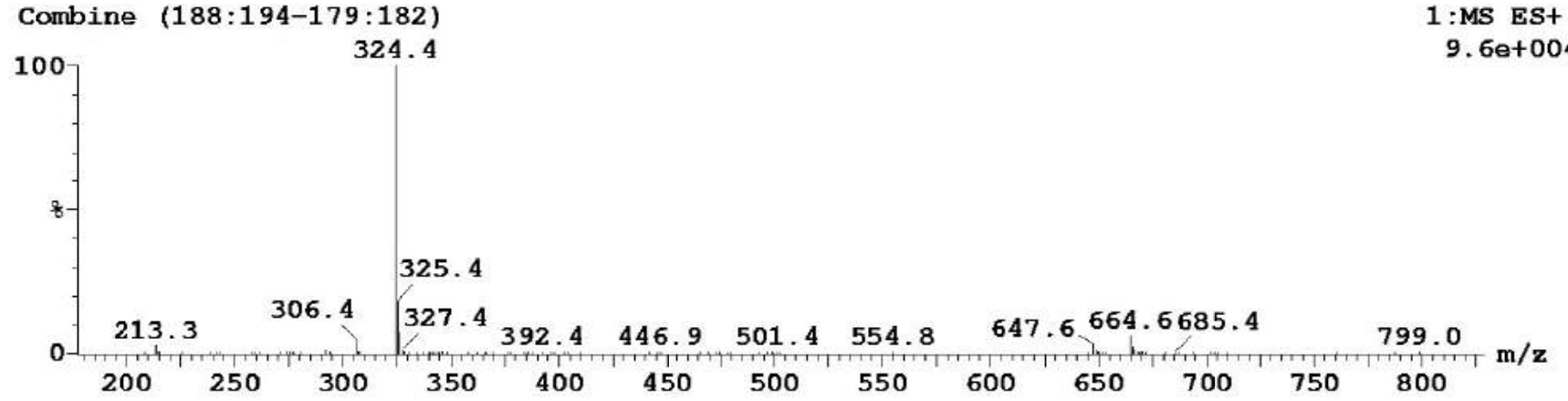


2: UV Detector: TIC

(2)

290.13

3.49

$1.095 e+2$

Range: $1.093 e+2$

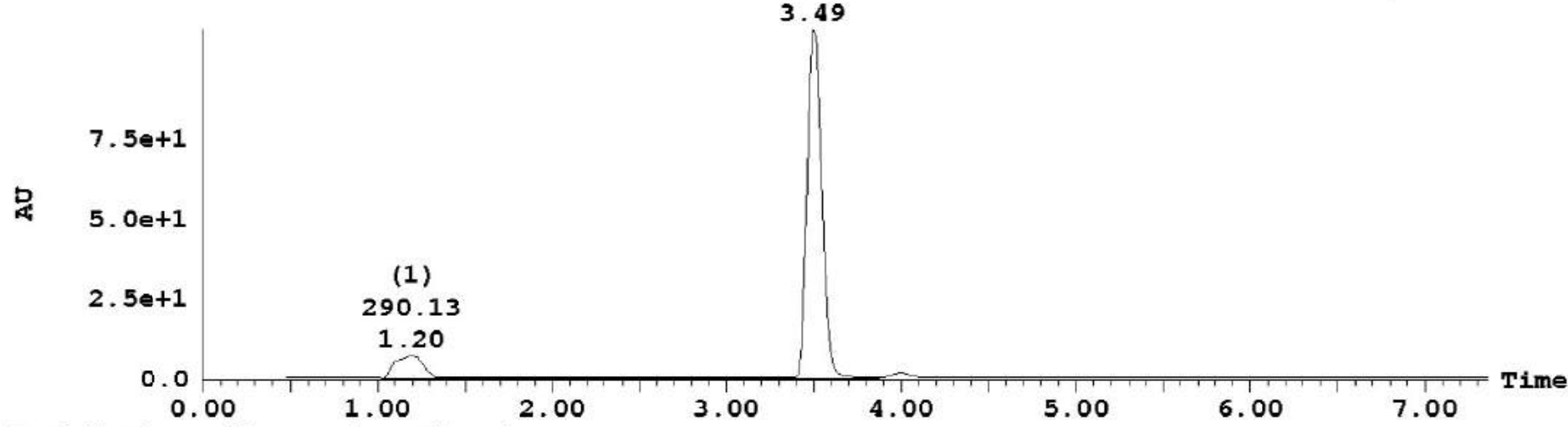

Peak Number Tim

Area \%Total

$\begin{array}{lll}1 & 1.20 & 10.99\end{array}$

$\begin{array}{lll}2 & 3.50 & 89.01\end{array}$

$\begin{array}{rrr}\text { Peak ID } & \text { Time } & \text { Mass Found } \\ 1 & 1.20 & 290.1390 .95\end{array}$

Combine (61:67-48:52)

1 :MS ES+

1. $6 e+003$

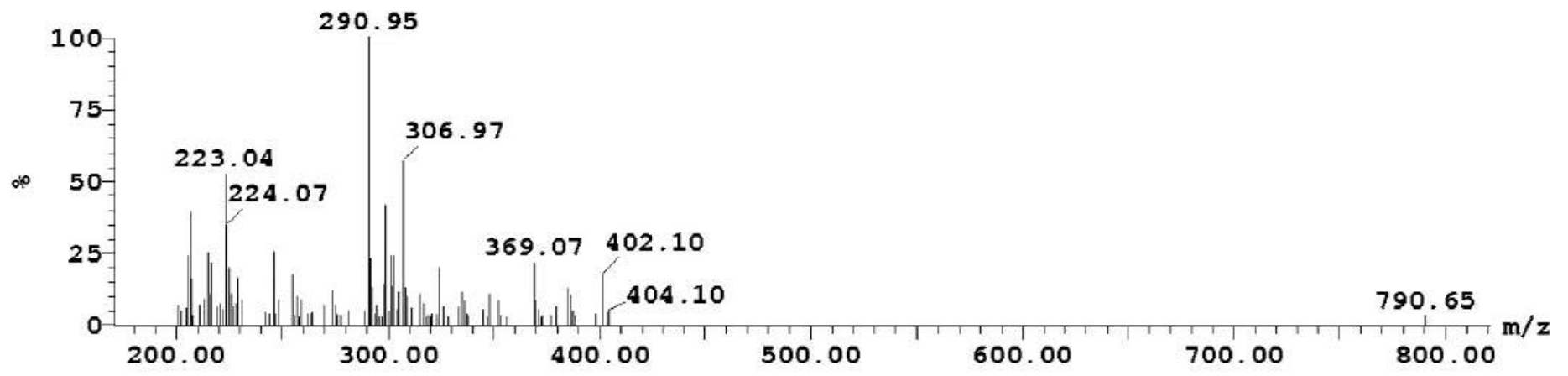

Peak ID Time Mass Found BPM

$2 \quad 3.50 \quad 290.1374 .14$

Combine (186:193-169:173)

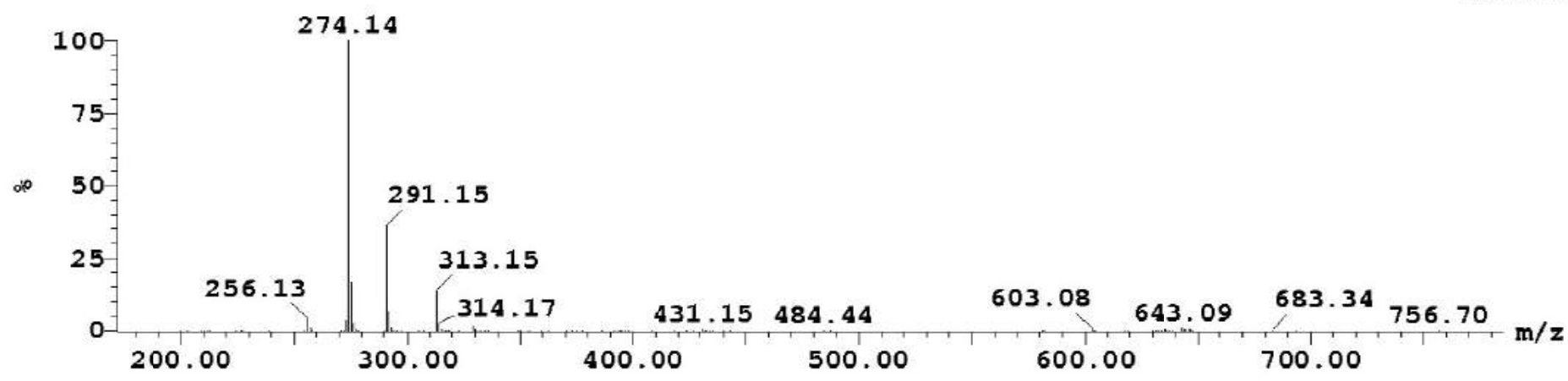


Printed: Wed May 01 09:54:11 2002

Sample Report (continued):

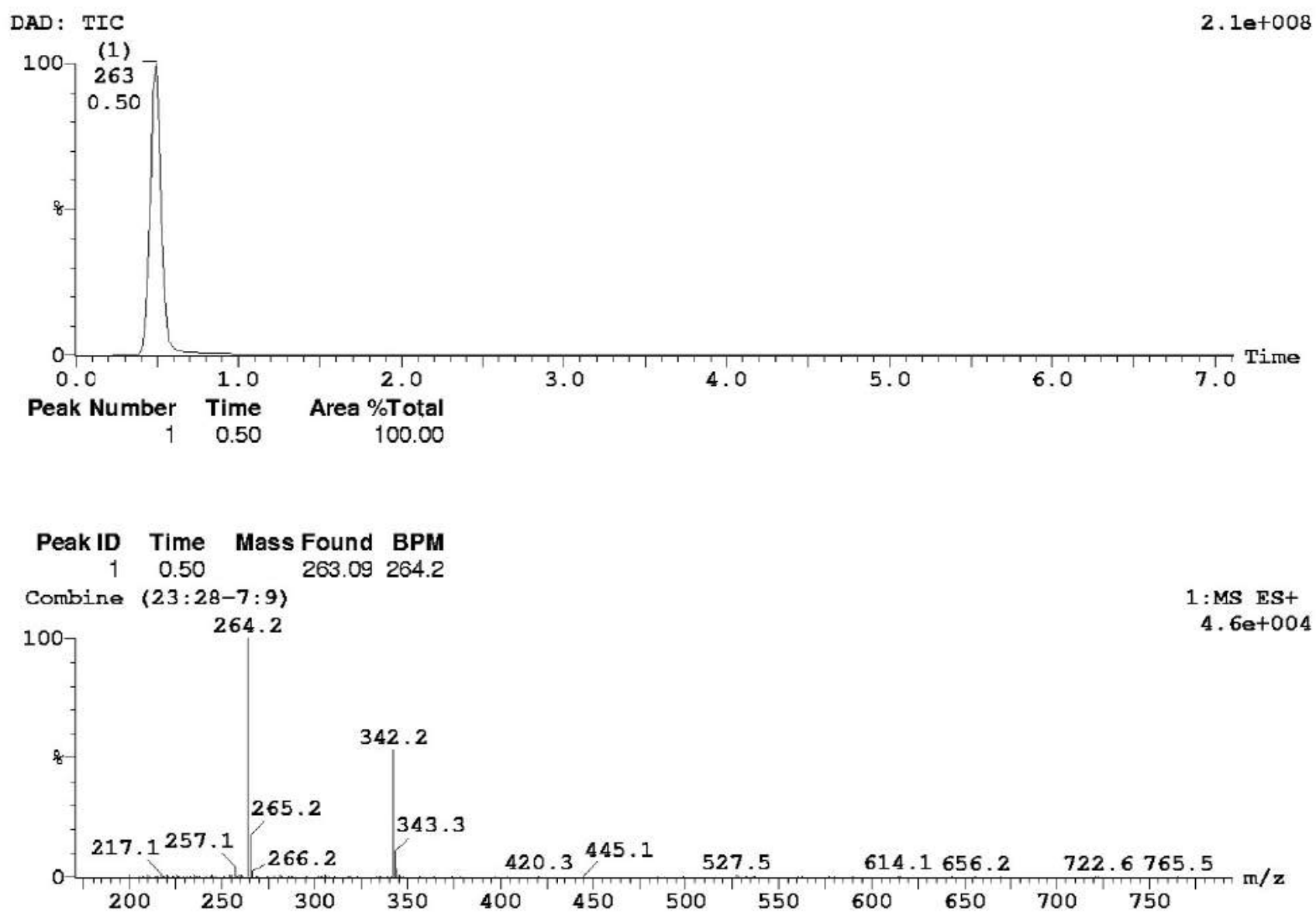


Printed: Thu Aug 01 17:05:11 2002

Sample Report (continued):

DAD: TIC

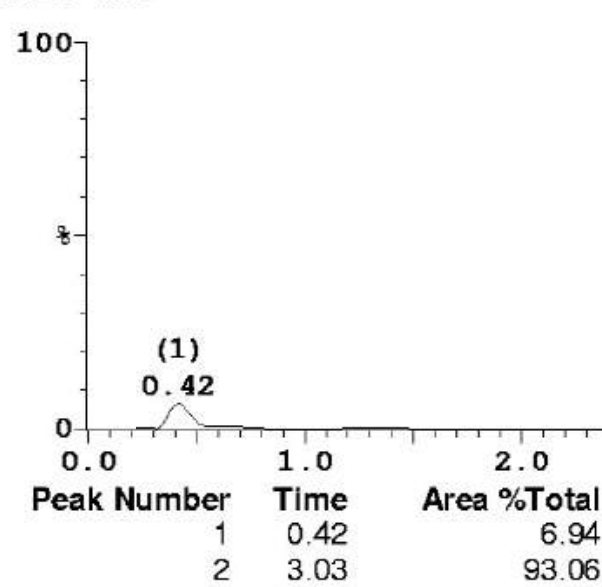

\section{1}

3.03
Time:06:31:43 
3: UV Detector: TIC

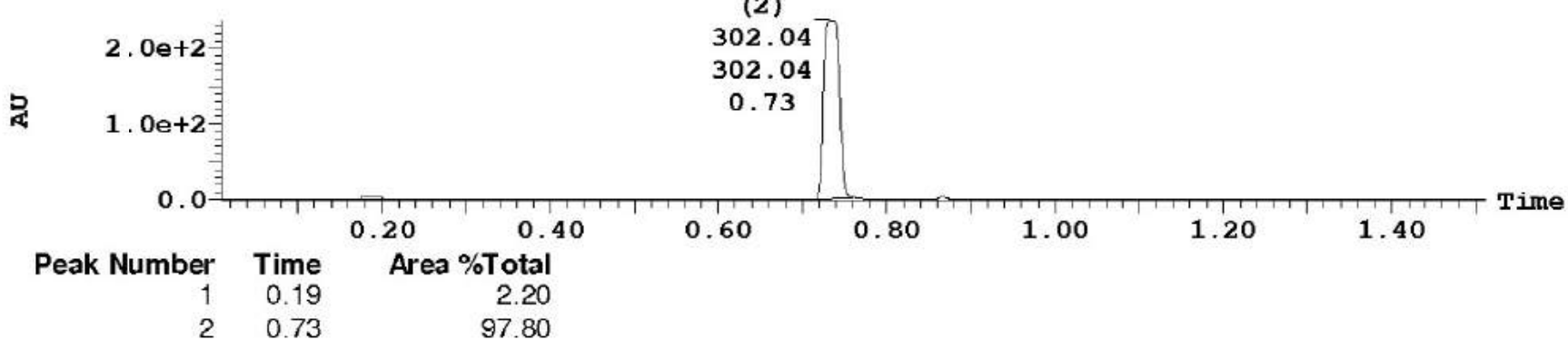

$\begin{array}{rrrrrrrr}\text { Peak ID } & \text { Time } & \text { Mass Found } & \text { BPM } & \text { Peak ID } & \text { Time } & \text { Mass Found } & \text { BPM } \\ 1 & 0.19 & & 157.00 & 1 & 0.19 & 180.93\end{array}$

1: (Time: 0.19)

1:MS ES+ 1: (Time: 0.19 )

2:MS ES-
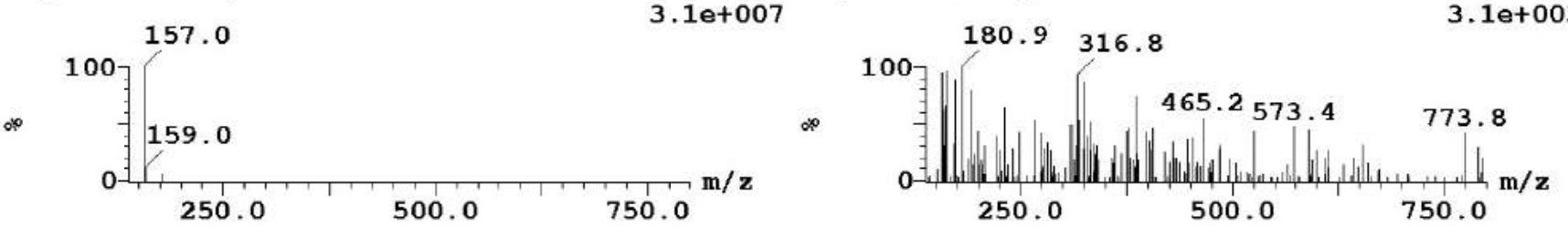

$\begin{array}{rrrr}\text { Peak ID } & \text { Time } & \text { Mass Found } & \text { BPM } \\ 2 & 0.73 & 303.04,, & 303.05\end{array}$

Peak ID Time Mass Found BPM

2 : (Time: 0.73 )

$1:$ MS ESt 2:(Time: 0.73 )

1. $2 \mathrm{e}+007$ $301.04 \quad 301.06$

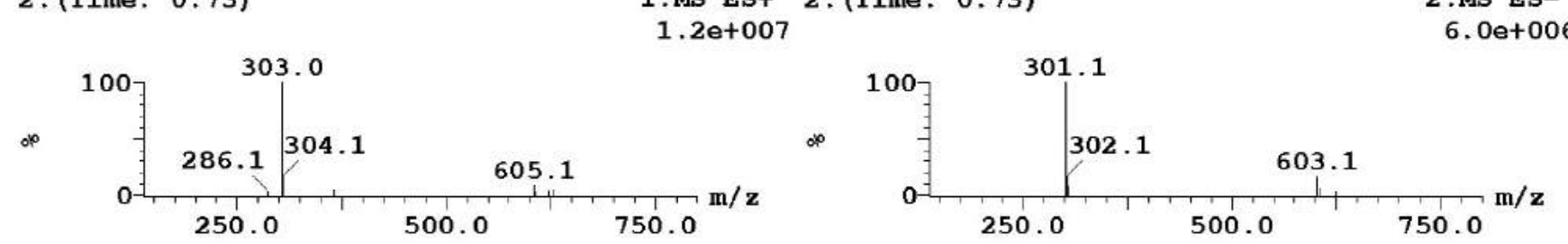




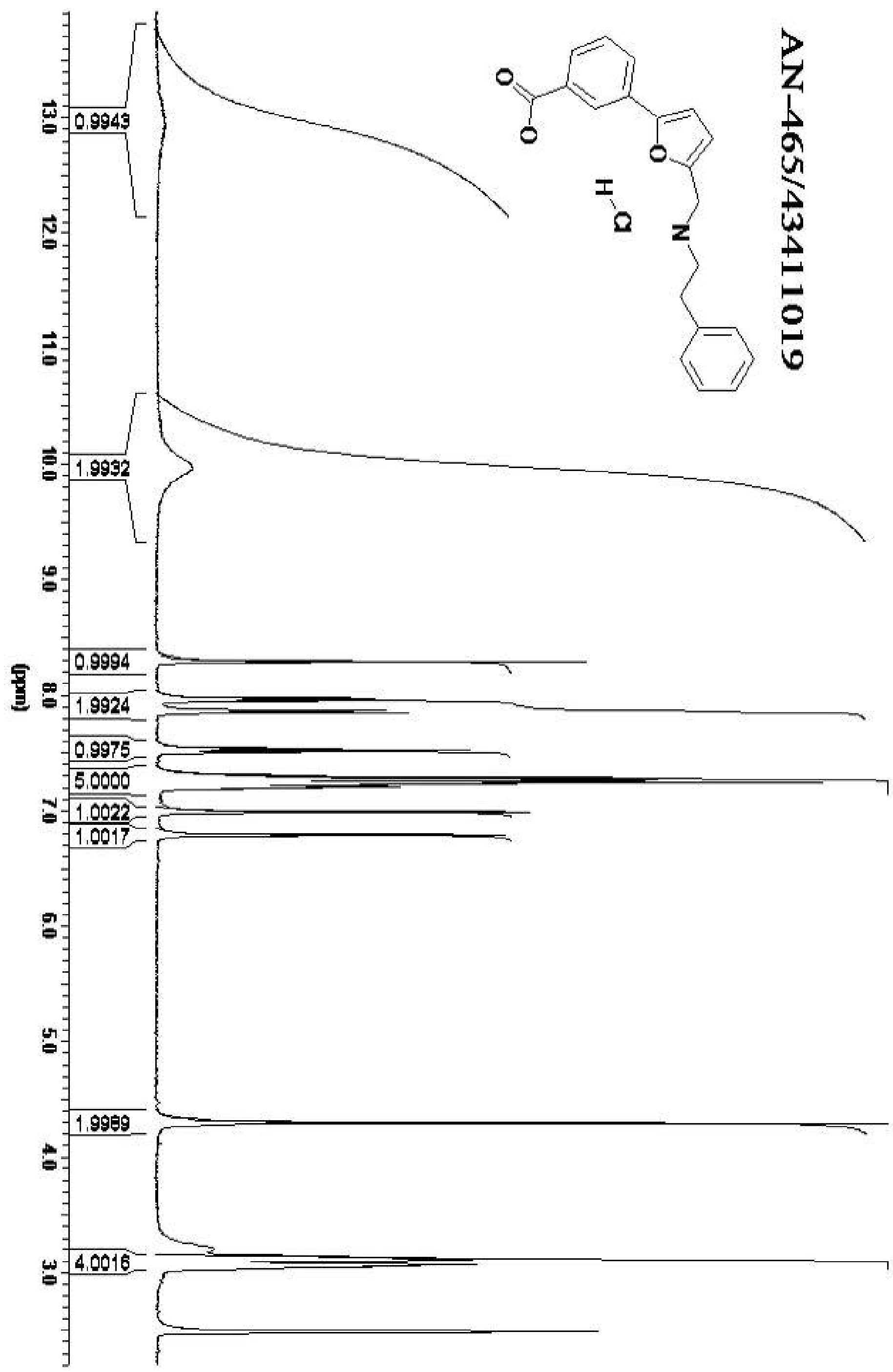




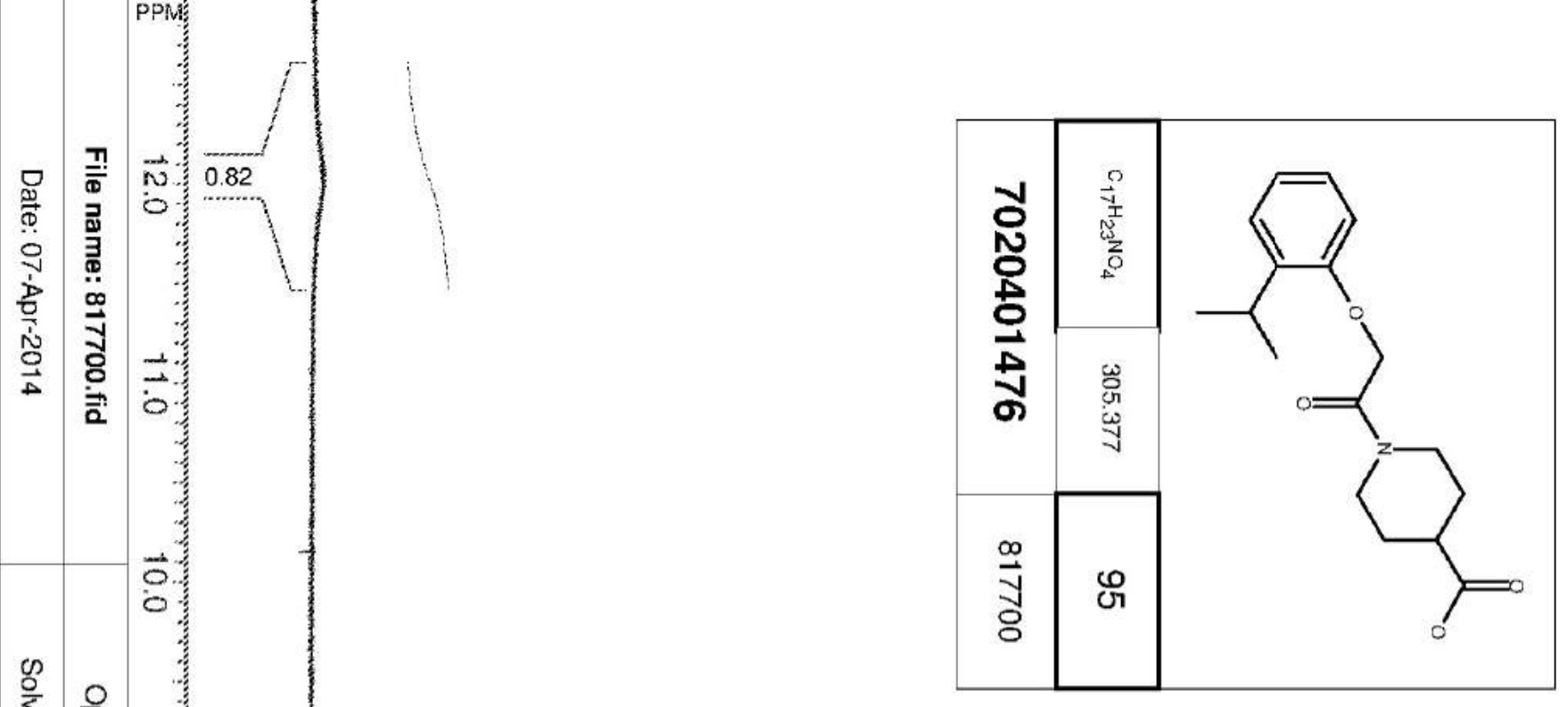

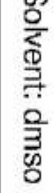

$\infty$

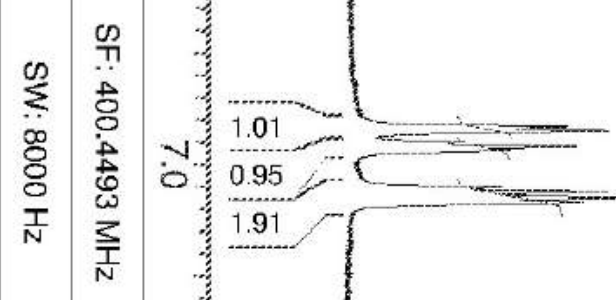

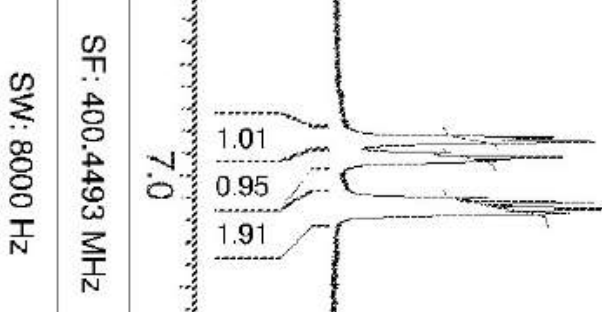

0

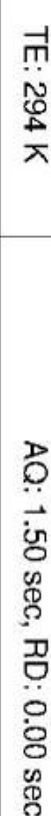

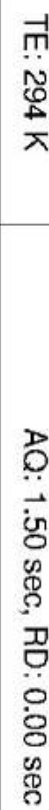

$\mathrm{c}$

12

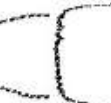

0.96

O

1.08

만

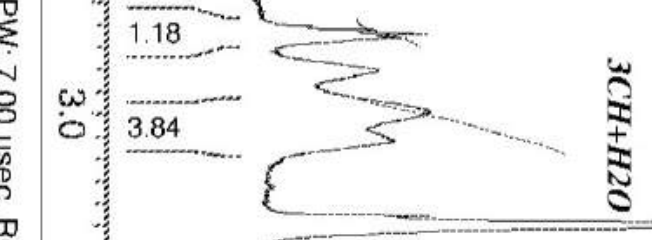

क $\mathrm{N}$

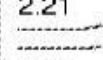

6.09

$\underset{0}{\stackrel{0}{0}}$ 


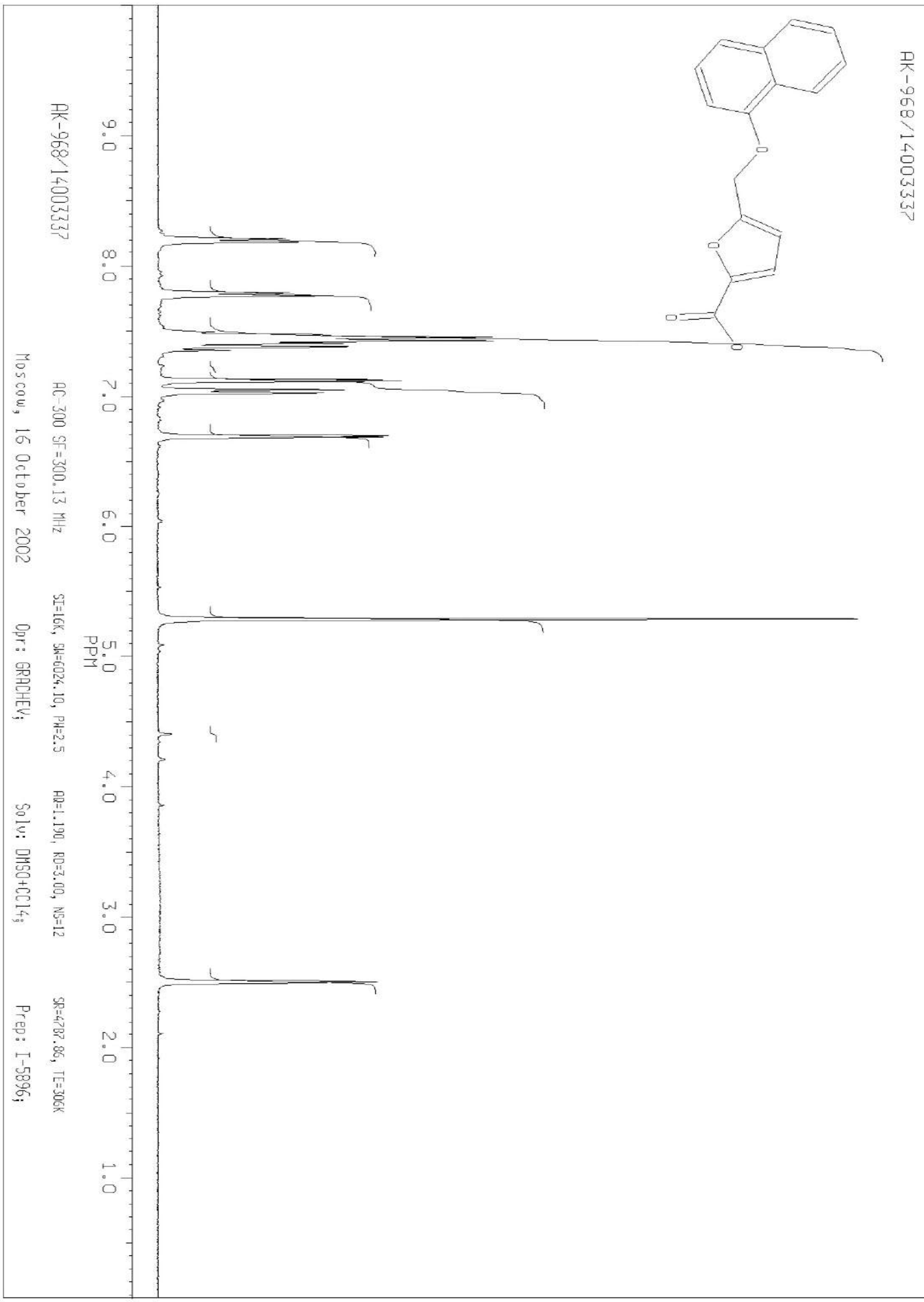




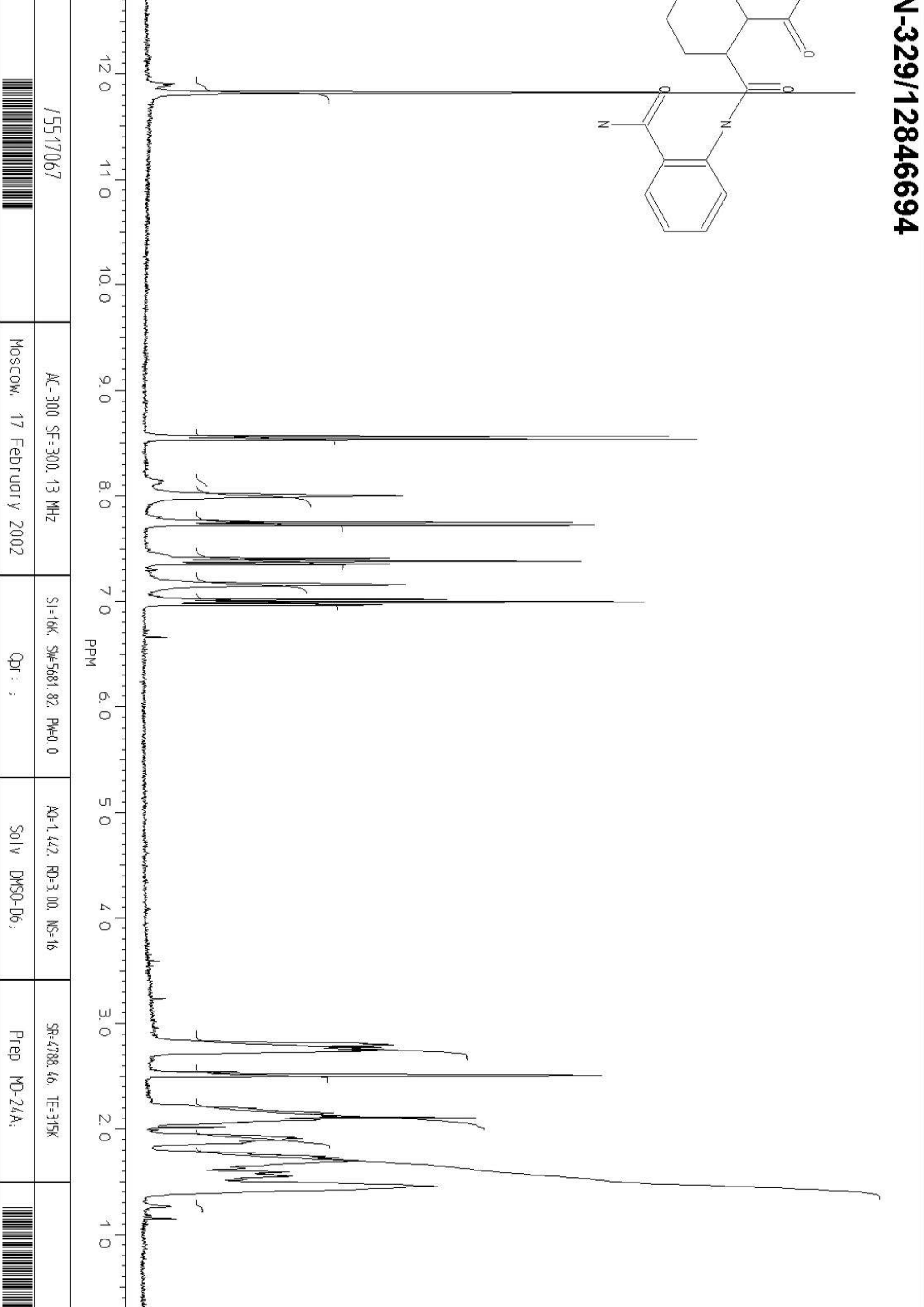




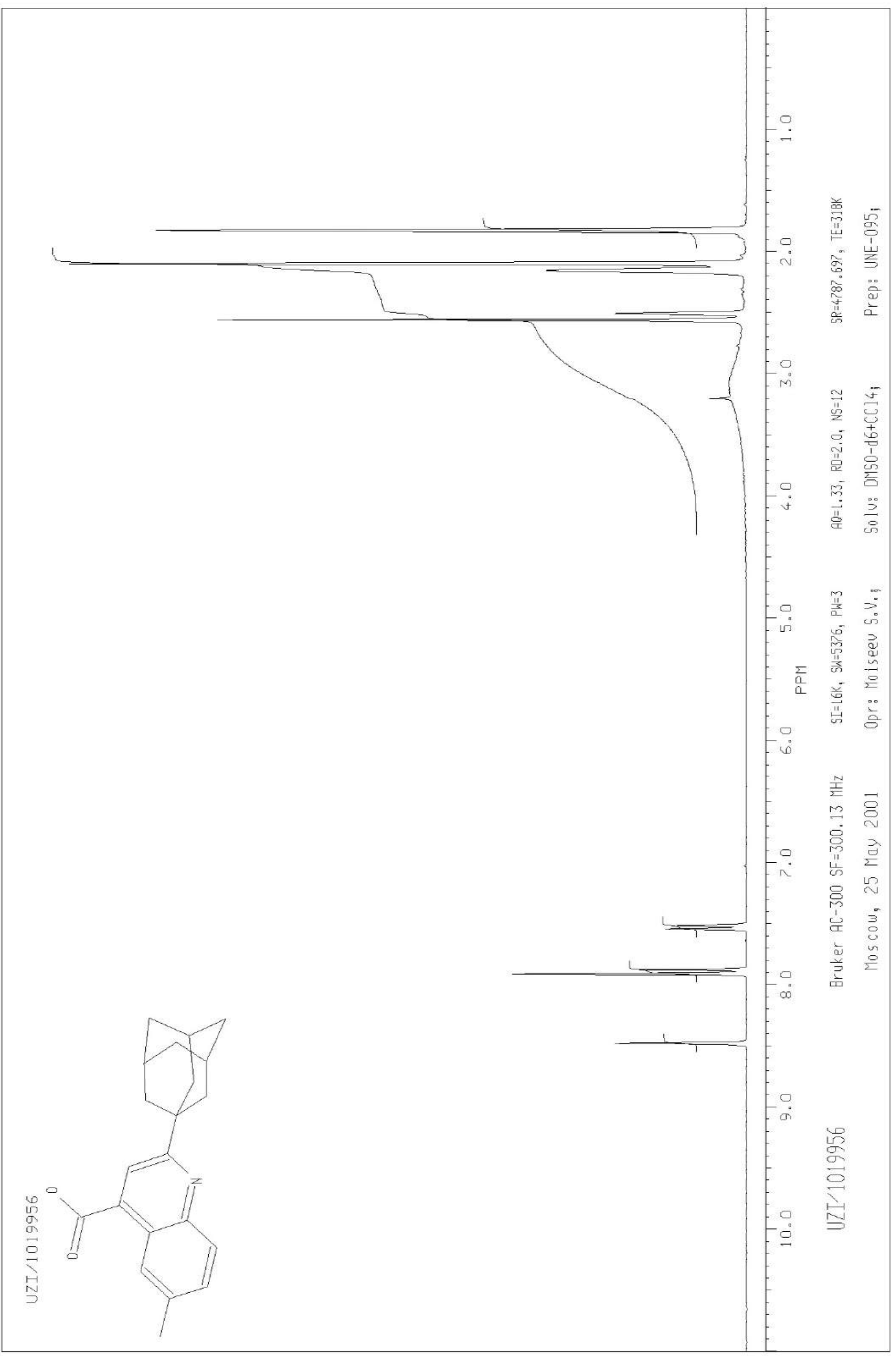




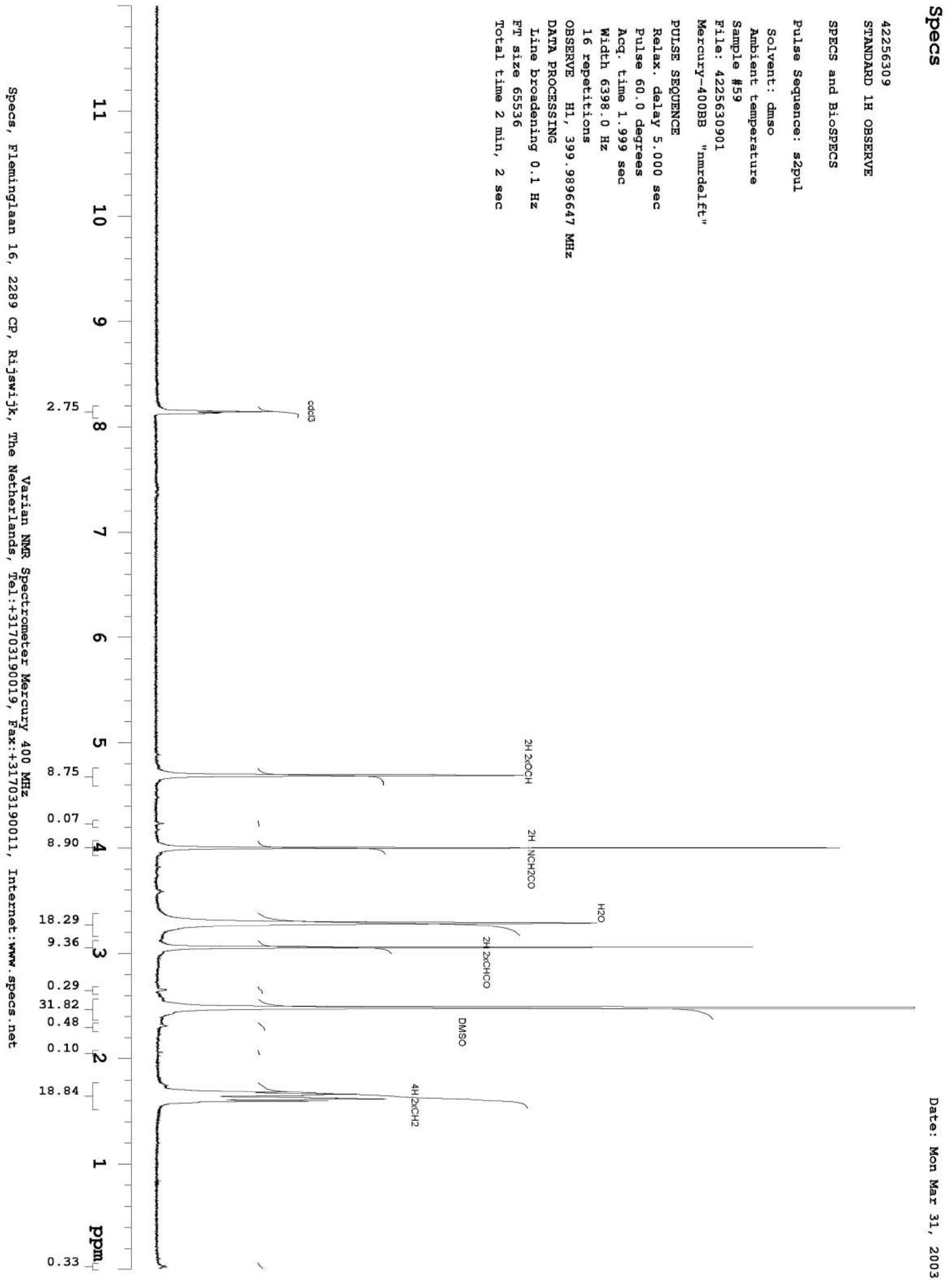




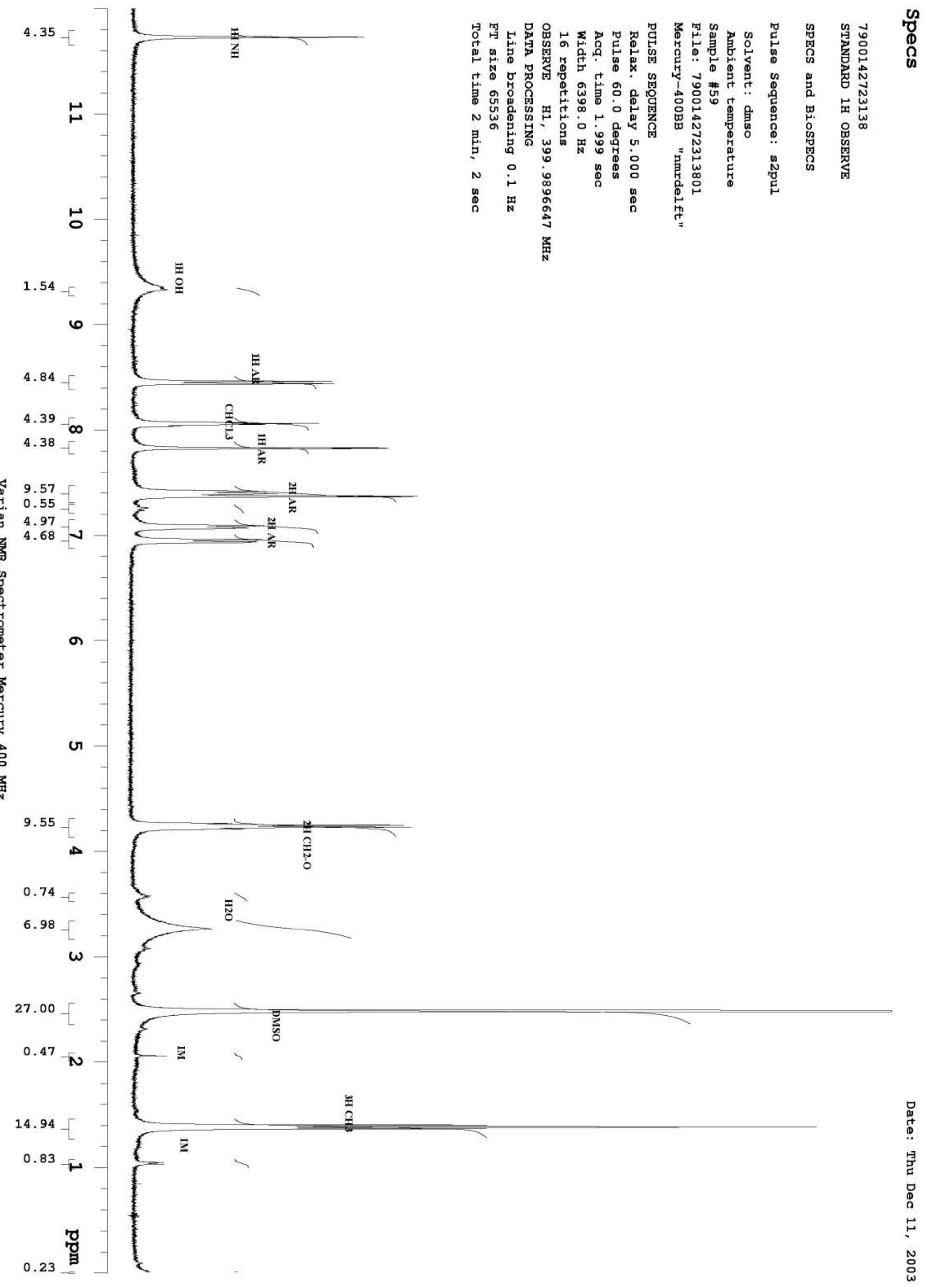




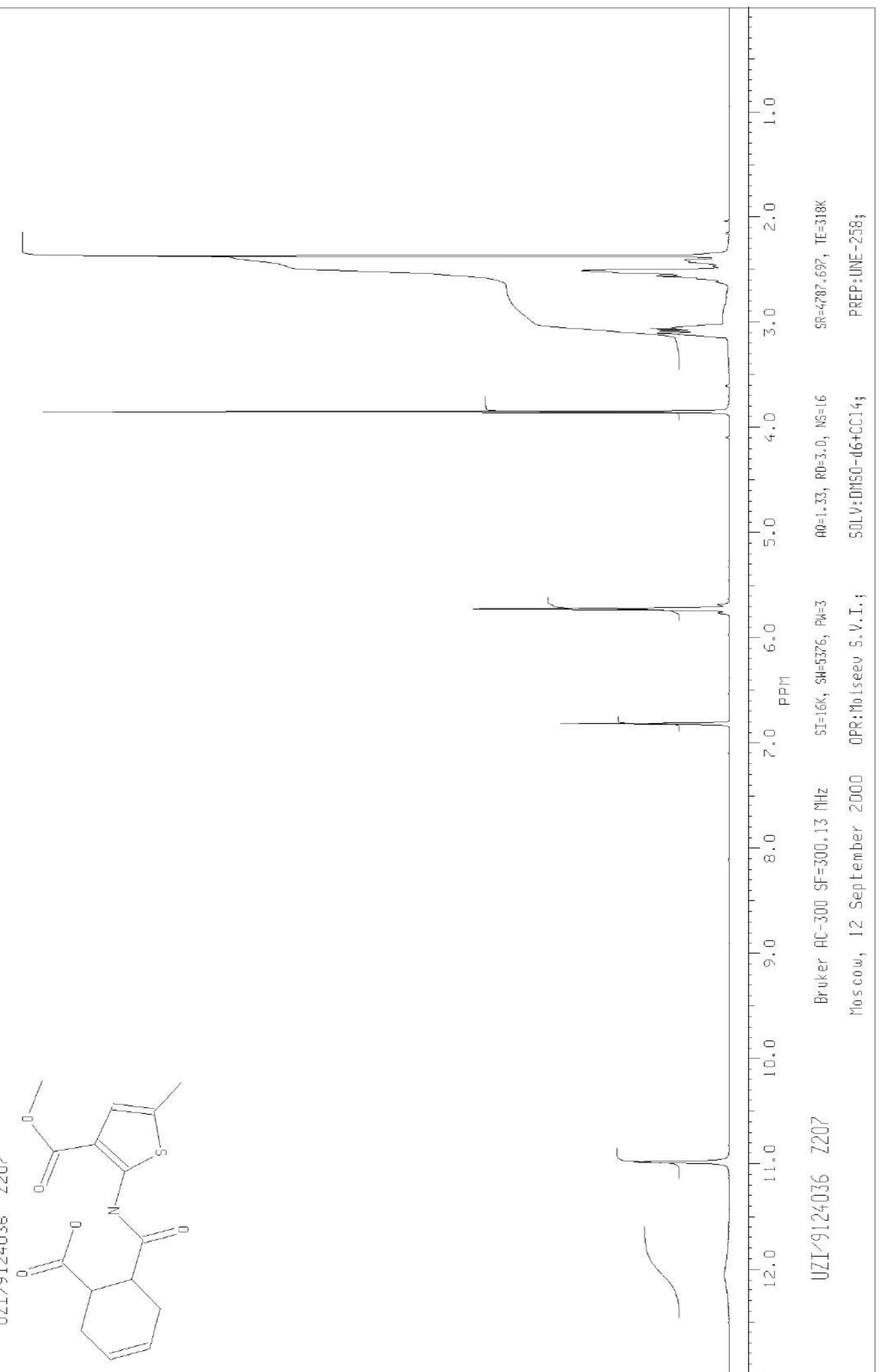




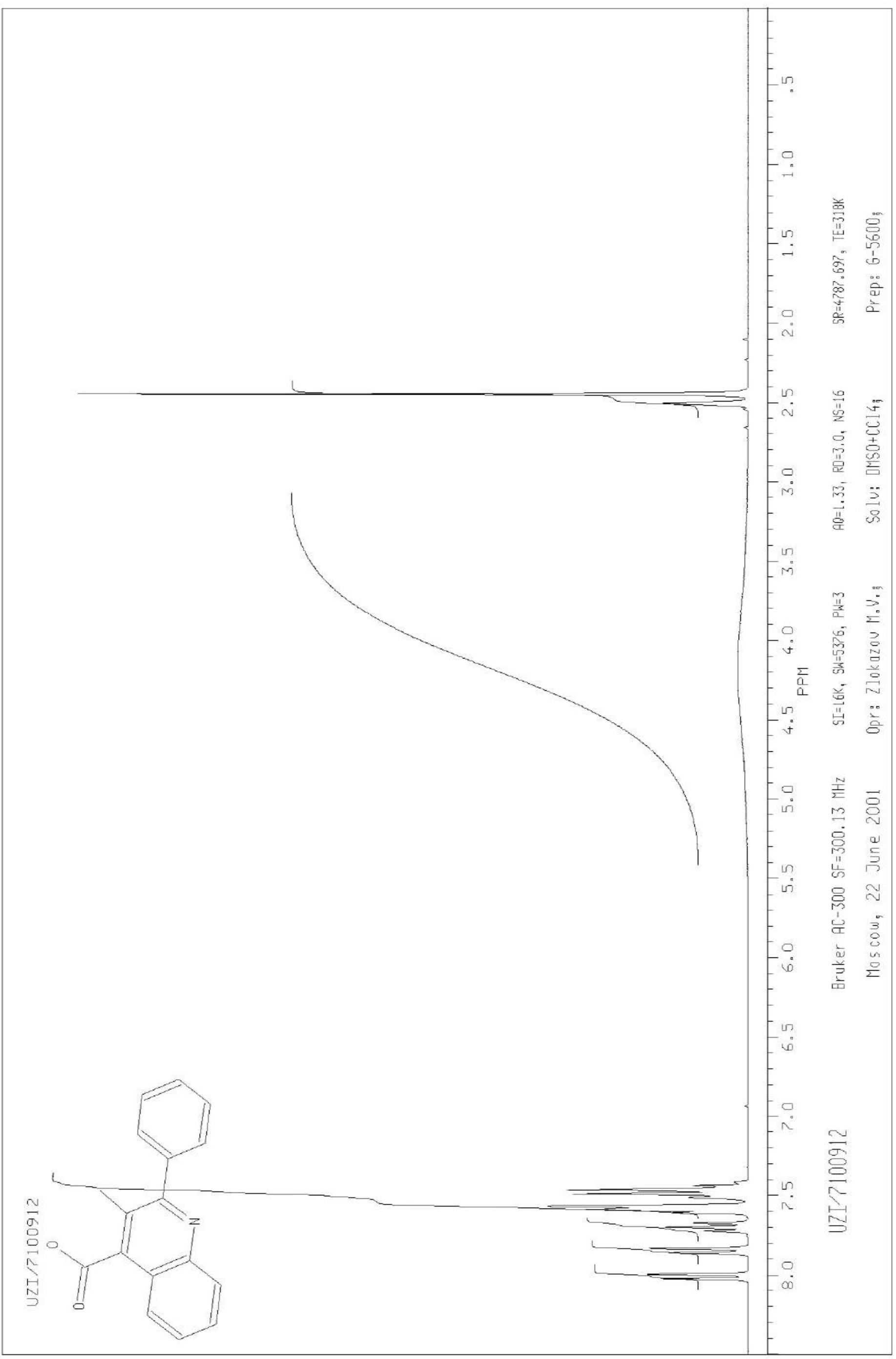


Specs

File:8115351100341

Instrument:System B

AQ-405/1100341

Vial:1:60

Printed: Fri May 30 15:39:30 2003

Sample Report (continued):

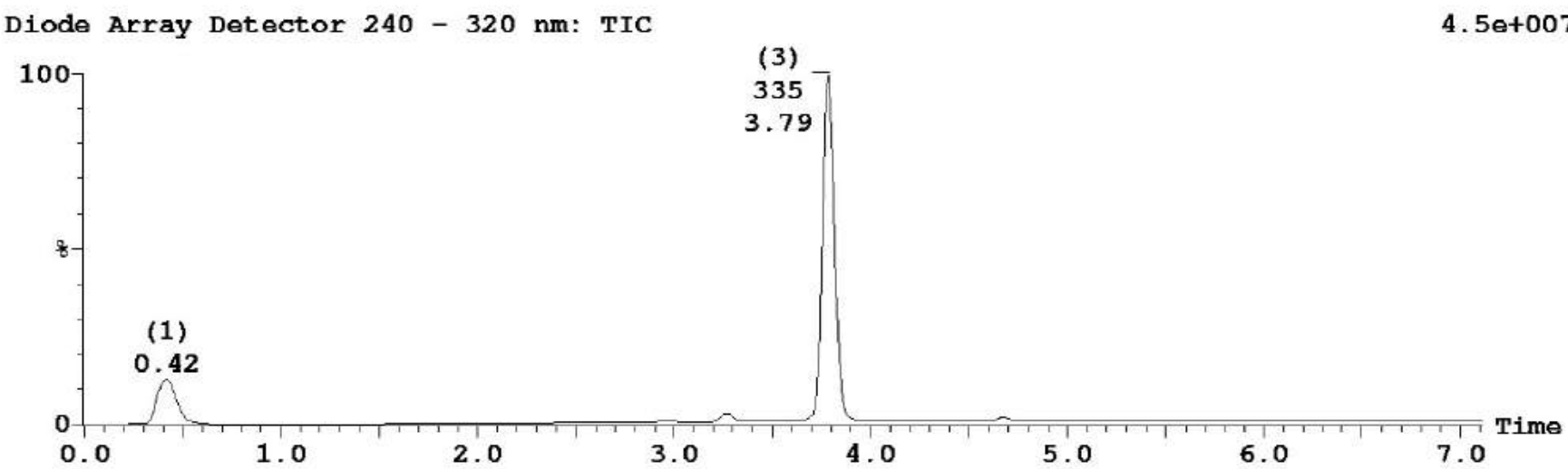

Peak Number Time Area \%Total

$\begin{array}{rrr}1 & 0.42 & 15.84 \\ 2 & 3.27 & 2.00 \\ 3 & 3.79 & 82.16\end{array}$

\section{Peak ID Time Mass Found BPM

$\begin{array}{llll}2 & 3.27 & 335.02 & 334\end{array}$

Combine (174:180-167:170)

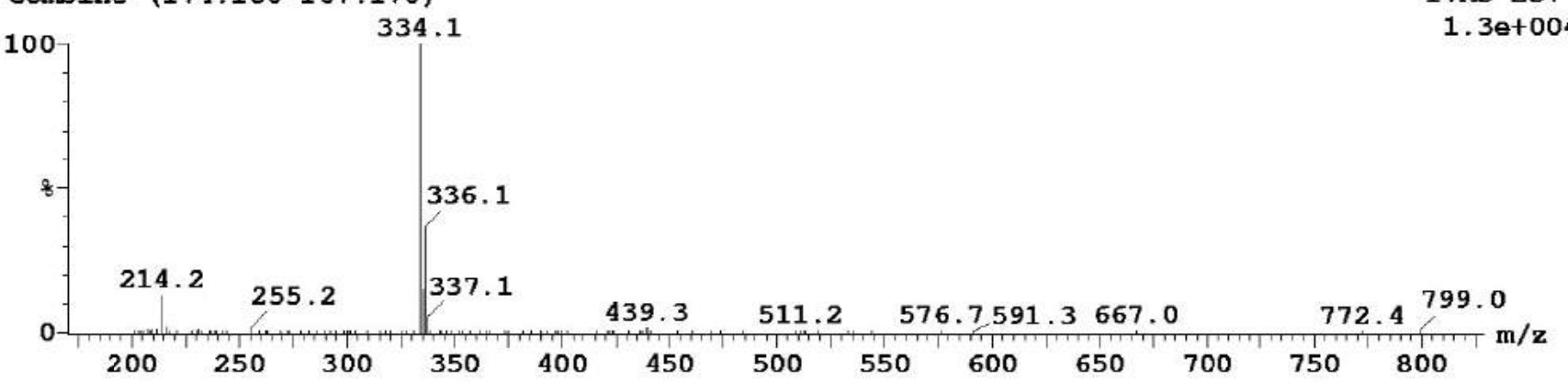

\section{Peak ID Time Mass Found BPM

$\begin{array}{llll}3 & 3.79 & 335.02 & 336\end{array}$

Combine (203:208-192:194)

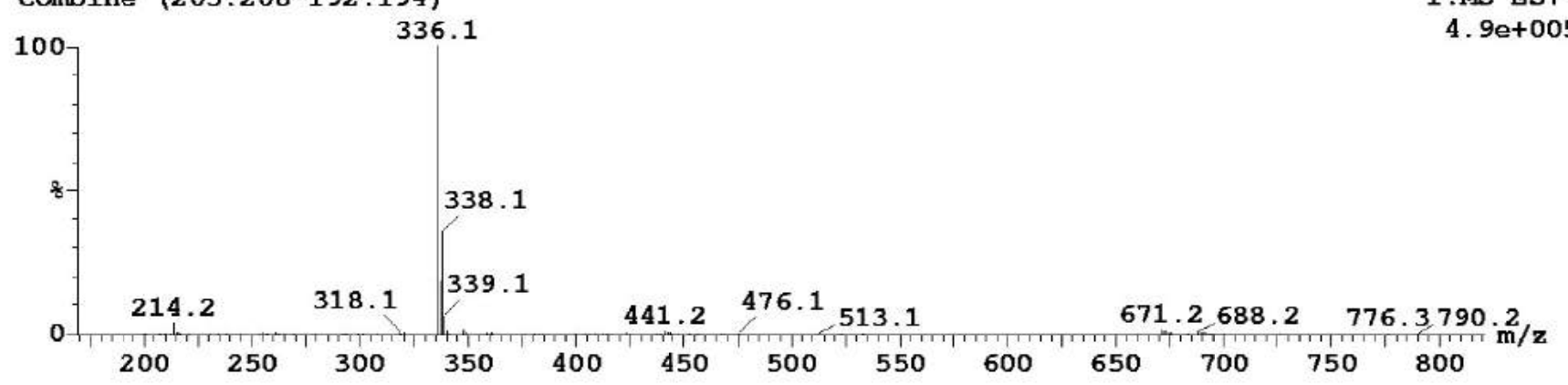

escription: $\mathrm{C} 16$

Date:26-Jun-2002 
3: UV Detector: TIC

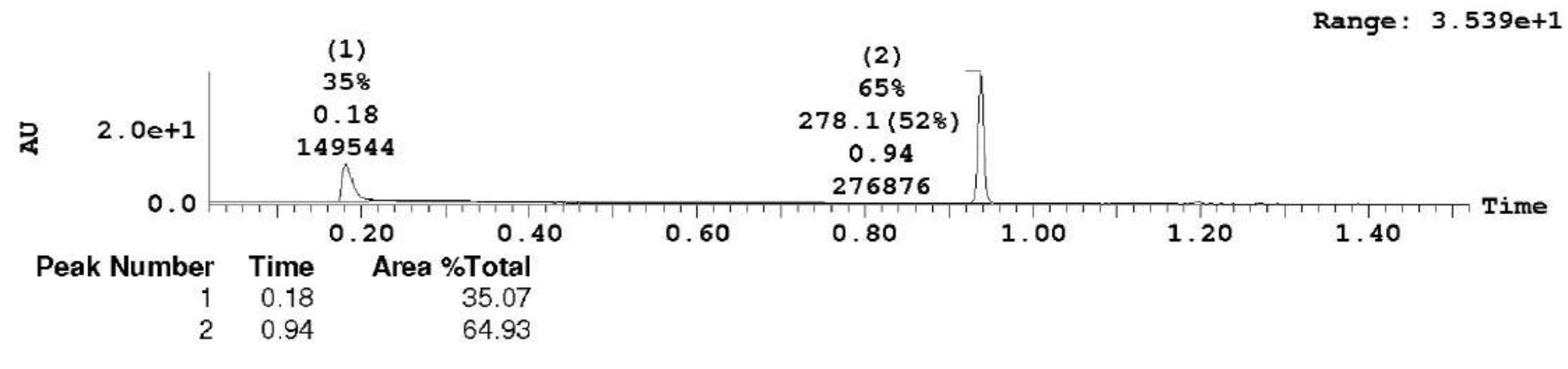

$$
\begin{array}{rr}
\text { Peak ID } & \text { Time } \\
1 & 0.18
\end{array} \text { Mass Found } \begin{array}{r}
\text { BPM } \\
145.89
\end{array}
$$

Peak ID Time Mass Found BPM

1: (Time: 0.18 )

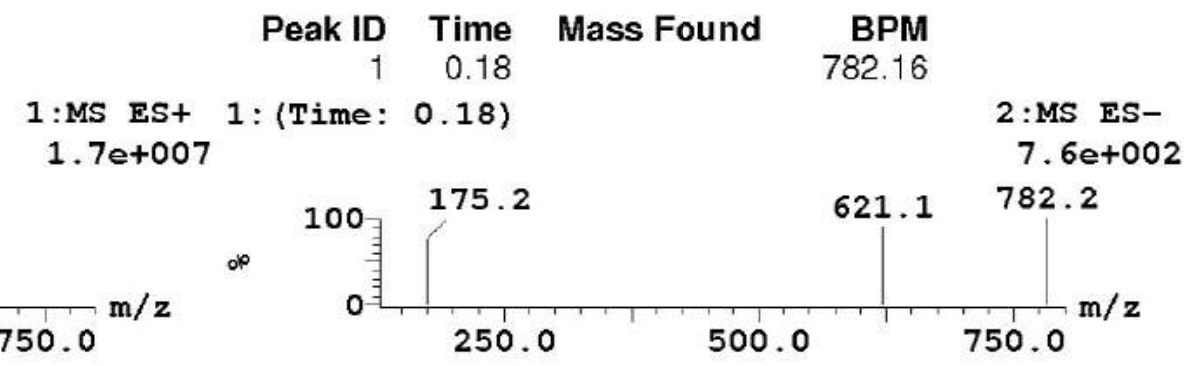
Peak ID Time Mass Found BPM 140.94

$2 \quad 0.94$
Peak ID Time Mass Found BPM
Found
278.09
277.17

2: (Time: 0.94 )

$\begin{array}{rr}\text { Peak ID } & \text { Time } \\ 2 & 0.94 \\ \text { 1:MS ES+ } \quad 2:(\text { Time: } & 0.94)\end{array}$

4. 0 e +006
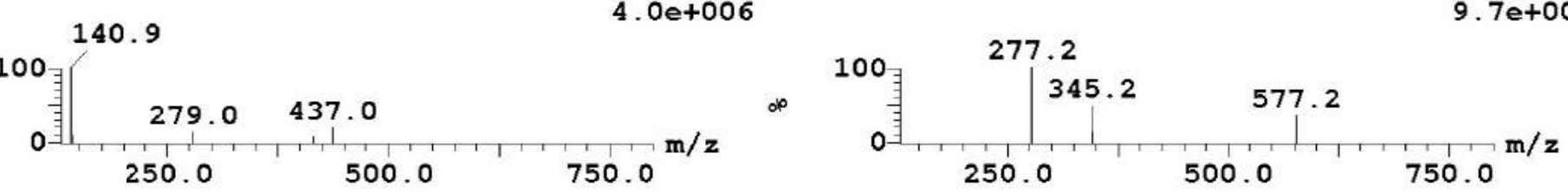
Printed: Fri Oct 26 16:01:53 2001

Sample Report (continued):

DAD: TIC

$$
100
$$

(0.0
0.41

Peak Number Time

$1 \quad 0.41$

23.81

7.83

92.17

8. $2 e+007$

(2)

321

3.81

\section{Peak ID Time Mass Found BPM}

$10.41 \quad 257.0$

Combine $(18: 24-12: 15)$

$1:$ MS ES+

$100 \quad 257.3$

1. $7 \mathrm{e}+003$

100

254.3

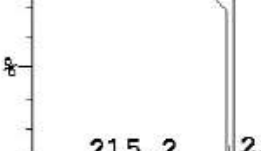

\begin{tabular}{l|ll}
215.2 & 259.3319 .2343 .2 & 397.
\end{tabular}

331.6 $\begin{array}{llll}567.3583 .2 & 662.3 & 692.9 & 768.7\end{array}$

200

250

$400 \quad 450$

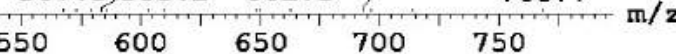

Peak ID Time Mass Found BPM

$2 \quad 3.81 \quad 321.17322 .0$

Combine (204:209-188:191)

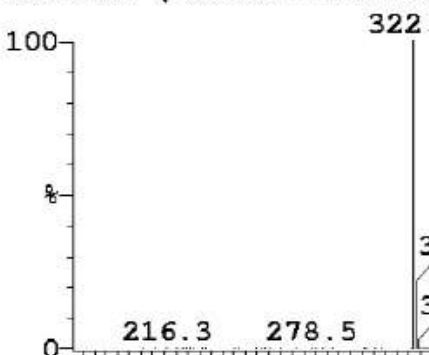

323.5

$\begin{array}{llllllll}324.5 & 400.5 & 428.2 & 528.3 & 611.2 & 643.5 & 716.4757 .7 & 788.7\end{array}$ $\begin{array}{lllllllllllllll}200 & 250 & 300 & 350 & 400 & 450 & 500 & 550 & 600 & 650 & 700 & 750 & 800 & \mathrm{~m} / \mathrm{z}\end{array}$ 
SPECS and BioSPECS B.V., The Netherlands

File:9900141204433

Vial:2:67

ID:AK-918/41204433

Date:22-Jun-2002

Printed: Wed Jun 26 16:33:32 2002

Sample Report (continued):

Description:C16 $15 \mathrm{NO}$

Time:16:35:08
DAD : TIC

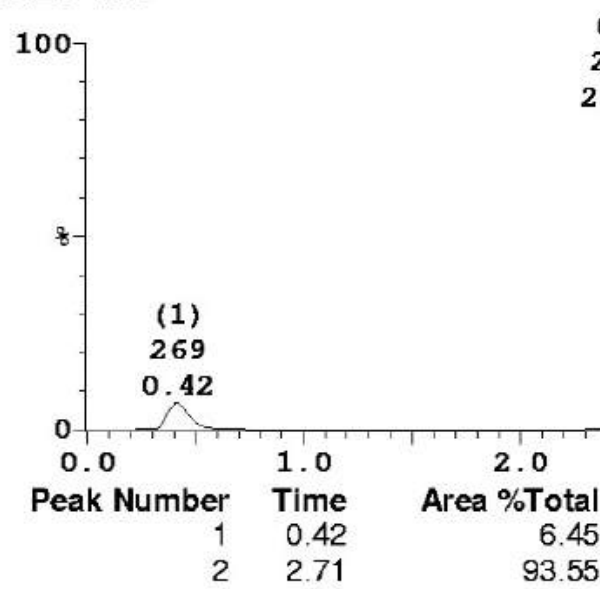

1. $3 e+008$

(2)

269

.71

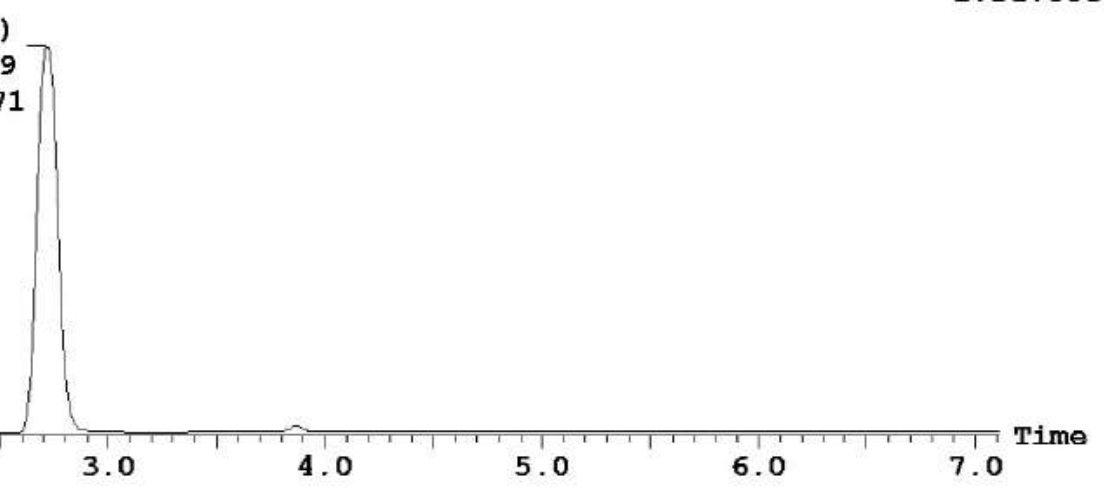

.55

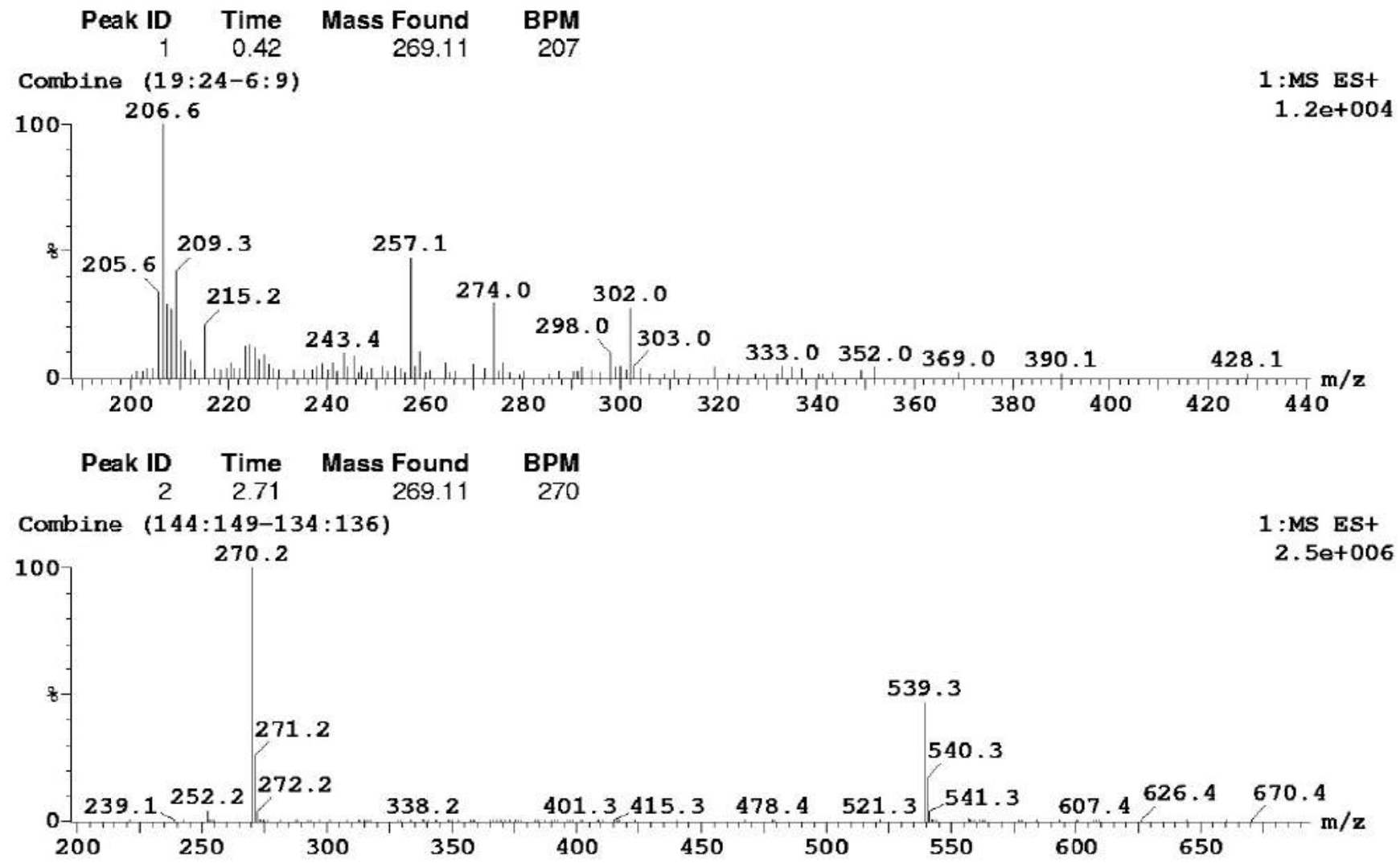

